\title{
Leptin-a mediates transcription of genes that participate in central endocrine and phosphatidylinositol signaling pathways in 72-hour embryonic zebrafish (Danio rerio)
}

\author{
Matthew Tuttle ${ }^{1}$ ， Mark R Dalman ${ }^{2}{ }^{\text {, }}$ Qin Liu ${ }^{1}$, Richard L Londraville ${ }^{\text {Corresp. } 1}$ \\ 1 Biology, University of Akron, Akron, $\mathrm{OH}$, United States \\ 2 Podiatric Medicine, Kent State University, Kent, $\mathrm{OH}$, United States \\ Corresponding Author: Richard L Londraville \\ Email address: Iondraville@uakron.edu
}

We analyzed microarray expression data to highlight biological pathways that respond to embryonic zebrafish Leptin-a (lepa) signaling. Microarray expression measures for 26,046 genes were evaluated from lepa morpholino oligonucleotide "knockdown", recombinant Leptin-a "rescue", and uninjected control zebrafish at 72-hours post fertilization. In addition to KEGG pathway enrichment for phosphatidylinositol signaling and neuroactive ligand-receptor interactions, Gene Ontology (GO) data from lepa rescue zebrafish include JAK/STAT cascade, sensory perception, nervous system processes, and synaptic signaling. In the zebrafish lepa rescue treatment, we found changes in the expression of homologous genes that align with mammalian leptin signaling cascades including AMPK (prkaa2), ACC (acacb), $\mathrm{Ca}^{2+} /$ calmodulin-dependent kinase (camkk2), PI3K (pik3r1), Ser/Thr protein kinase B (akt3), neuropeptides (agrp2, cart1), mitogen-activated protein kinase (MAPK), and insulin receptor substrate (LOC794738, LOC100537326). Notch signaling pathway and ribosome biogenesis genes respond to knockdown of Leptin-a. Differentially expressed transcription factors in lepa knockdown zebrafish regulate neurogenesis, neural differentiation, and cell fate commitment. This study presents a role for zebrafish Leptin-a in influencing expression of genes that mediate phosphatidylinositol and central endocrine signaling. 
1

2 Leptin-a mediates transcription of genes that participate in central endocrine and phosphatidylinositol signaling pathways in 72-hour embryonic zebrafish (Danio rerio).

7 1Biology, University of Akron, Akron, OH, United States

8 2Podiatric Medicine, Kent State University, Kent, $\mathrm{OH}$, United States

10 *Corresponding Author: Richard Londraville rlondra@uakron.edu

$11 \quad 330-972-7151$

$12 \quad 330-972-8445$ (Fax)

13 Department of Biology

Doctoral Program in Integrated Bioscience

Auburn Science and Engineering Center, C310

Akron, $\mathrm{OH}, 44325-3908$ supplementary file (.xIsx). 


\section{Abstract}

We analyzed microarray expression data to highlight biological pathways that respond to embryonic zebrafish Leptin-a (lepa) signaling. Microarray expression measures for 26,046 genes were evaluated from lepa morpholino oligonucleotide "knockdown", recombinant Leptin-a "rescue", and uninjected control zebrafish at 72-hours post fertilization. In addition to KEGG pathway enrichment for phosphatidylinositol signaling and neuroactive ligand-receptor interactions, Gene Ontology (GO) data from lepa rescue zebrafish include JAK/STAT cascade, sensory perception, nervous system processes, and synaptic signaling. In the zebrafish lepa rescue treatment, we found changes in the expression of homologous genes that align with mammalian leptin

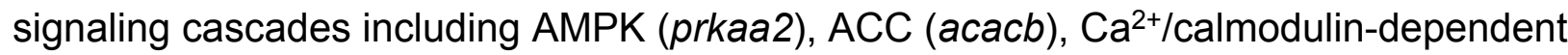
kinase (camkk2), PI3K (pik3r1), Ser/Thr protein kinase B (akt3), neuropeptides (agrp2, cart1), mitogen-activated protein kinase (MAPK), and insulin receptor substrate (LOC794738, LOC100537326). Notch signaling pathway and ribosome biogenesis genes respond to knockdown of Leptin-a. Differentially expressed transcription factors in lepa knockdown zebrafish regulate neurogenesis, neural differentiation, and cell fate commitment. This study presents a role for zebrafish Leptin-a in influencing expression of genes that mediate phosphatidylinositol and central endocrine signaling.

\section{Introduction}

In humans and rodents, the absence of a functional leptin (ob/ob) or leptin receptor $(d b / d b)$ causes hyperphagia, early-onset and morbid obesity, severe type II diabetes, hypogonadotropic hypogonadism, impaired thermogenesis, and dysregulation of bone growth and immune response (Friedman and Halaas, 1998). Leptin (Iep), a conserved $16 \mathrm{kDa}$ pleiotropic peptide hormone, is primarily secreted from adipocytes in proportion to fat mass (Maffei et al., 1995; Zhang et al., 1994). Leptin receptor (lepr) has 6 isoforms in mammals that vary in cytoplasmic domain length, tissue distribution, and signaling competence (Chen et al., 1996; Lee et al., 1996). The leptin receptor long isoform $(\mathrm{Ob}-\mathrm{Rb})$ is expressed in the hypothalamus and interacts with members of the Janus kinase (JAK) and signal transducers and activators of transcription (STAT) protein families. Leptin signaling in mammals mediates anorectic (CART, POMC) and 
52 orexigenic (AgRP, NPY) circuits in the arcuate nucleus as well as voltage-gated calcium 53 channels in the lateral hypothalamus (Baskin et al., 1999; Jo et al., 2005; Park and Ahima, 2014; Schwartz et al., 1997). ob/ob, and $d b / d b$ obese rodent mutants, and dietinduced obesity mammals have reduced STAT-3 signaling in the arcuate nucleus which is generally attributed to either an absence of the hormone, an inability to respond to the hormone, or central leptin resistance (Ghilardi et al., 1996; Münzberg et al., 2004; Vaisse et al., 1996). Tissue-specific deletion of Ob-Rb in rodent neurons, but not hepatocytes, results in obesity, indicating that the regulatory effects of leptin signaling on adipose mass are mediated centrally (Cohen et al., 2001). Leptin supplementation rescues morbid phenotypes associated with ob/ob but not with $d b / d b$ rodents which agrees with parabiosis experiments (Coleman, 1973); these findings mimic the effects of recombinant leptin in le ${ }^{-/}$and lepr/- human case studies (Farooqi et al., 1999, 2002, 2007; Gibson et al., 2004; Montague et al., 1997; Pelleymounter et al., 1995).

Human leptin regulates JAK/STAT, AMPK/ACC, PI3K/Akt/FOXO1, and SHP2/MAPK intracellular signal transduction cascades (Park and Ahima, 2014). Extracellular signals transduced through leptin and insulin receptors (IR) in neuron populations of the hypothalamus stimulate IRS/PI3K/Akt/FOXO1 pathways (Park and Ahima, 2014; Xu et al., 2005a). Leptin-dependent regulation of AMP-activated protein kinase (AMPK) and phosphoinositide 3-kinase (PI3K) activity in the arcuate nucleus also mediates liver homeostasis through hypothalamic-autonomic circuits in mammals (Tanida et al., 2015). Central leptin signaling is mitigated by negative feedback loops that involve SOCS3, which is transactivated by STAT-3, as well as PTP1B (Dunn et al., 2005; Park and Ahima, 2014). Peripheral leptin signaling in mammals activates lipid metabolism through the regulation of AMPK and acetyl coenzyme A carboxylase (ACC) (Minokoshi et al., 2002).

Are the pathways stimulated by mammalian leptin signaling conserved across vertebrates? Genomes from all vertebrate classes have at least one copy of leptin (Londraville et al., 2017). Despite low sequence homology, fish leptin genes share a syntenic relationship with human lep (Gorissen et al., 2009; Howe et al., 2013; Kurokawa et al., 2005), and the zebrafish genome has orthologous members of other cytokine and receptor families including IFN-I (interferon type I), IFN-II (interferon type- 
83 II), IL (interleukins), chemokines, and TNF (tumor necrosis factors) (Savan and Sakai, 84 2006). Although all mammals express single lep genes, many fishes express multiple

leptin paralogs (likely due to an ancestral teleost whole-genome duplication event; Jaillon et al., 2004). As opposed to single lep orthologues in humans and rodents, the impact of having multiple copies of leptin on fish physiology and gene regulation is not well-understood. Zebrafish lepa message is abundant in liver, and lepb expression is highest in ovary (Gorissen et al., 2009). The putative zebrafish lepb promoter contains $a \sim 1.3 \mathrm{~kb}$ enhancer element that responds to regeneration cues, and hepatic lepb expression is downregulated in response to fasting (Gorissen et al., 2009; Kang et al., 2016). Zebrafish lepa is induced by hypoxic cues in adult liver and embryonic muscle, presumably through hypoxia inducible transcription factor 1 (Chu et al., 2010). The induction of lepa and lepb expression in response to hypoxia and tissue regeneration, respectively, suggests that zebrafish leptins may integrate different responses to stress. The zebrafish genome has one leptin receptor (lepr) which has broad tissue expression including gill, liver, ovary, spleen, gut, heart, and pituitary (Gorissen et al., 2009; Liu et al., 2010). In contrast to mammalian $d b / d b$ analogues, adult zebrafish leptin receptor knockouts (leprsa1508) do not have differences in adiposity, feeding, fecundity, or body size compared to controls (Michel et al., 2016), unlike the response to lepr knockout in medaka (Chisada et al., 2014). Knockout leprsa1508 larvae have increased numbers of $\beta$-cells as well as upregulation of insulin-a and glucagon-a (which can be blocked by metformin) suggesting that peripheral leptin signaling accommodates glucose homeostasis, but not adipostasis in zebrafish (Michel et al., 2016). A more complete understanding of zebrafish Leptin-a and Leptin-b binding to the receptor, and their respective signaling cascades is needed to understand leptin function in fishes.

Stat-3 signaling stimulated by leptin has been documented for several fish species (Gong et al., 2016, Wu et al. 2016A,B, Douros et al., 2018) and frogs (Cui et al., 2014). For Xenopus (Cui et al., 2014) and Tilapia (Douros et al., 2018), large-scale transcriptomic response to leptin stimulation is established. These innovative studies add much to our understanding of the evolution of leptin response, but they do not measure transcriptomic response with reduced leptin signaling.

Zebrafish embryos (<1 hpf) injected with lepa morpholinos (lepa morphants) are 
114 verified to have reduced Leptin-a protein expression, developmental abnormalities

115 (reduced eye size, reduced otic vesicle formation, bent notochord), and reduced

116 metabolic rate, all of which are ameliorated by co-injection of recombinant zebrafish

117 Leptin-a (lepa 'rescue'; Liu et al., 2012, Dalman et al., 2013). In this study we used the

118 established lepa morphant model to investigate the role of Leptin-a in embryonic gene

119 regulation. RNA prepared from lepa morphants, lepa rescue, and control zebrafish

120 embryos was processed on single-channel microarrays; digital expression estimates

121 were generated for 26,046 unique gene symbol identifiers across 16 total samples.

122 Differentially expressed genes (DEG's), KEGG pathways, and Gene Ontologies (GO)

123 were evaluated for three pairwise comparisons: lepa morphants compared to uninjected

124 controls; lepa rescue compared to lepa morphant; and lepa rescue compared to

125 uninjected controls. We tested the null hypothesis that zebrafish Leptin-a mediates

126 energy homeostasis through central endocrine mechanisms analogous to human and

127 rodent leptins. We expected that the transcription of genes in adipocytokine and lipid

128 signaling, endocrine pathways, JAK/STAT cascades, glucose homeostasis, and

129 immune function respond to embryonic zebrafish lepa knockdown and recombinant

130 Leptin-a rescue treatments.

\section{Materials and Methods}

132

133

134

135

136

137

138

139

140

141

142

143

\subsection{Animal Care and Ethical Procedures: All zebrafish and associated animal} procedures were reviewed and approved by The University of Akron's Institutional Animal Care and Use Committee (IACUC, approval reference \# 08-6B). Adult zebrafish were obtained from Aquatic Tropicals (Bonita Springs, $\mathrm{FL}$ ), and maintained in aquatic fish housing systems at $28.5^{\circ} \mathrm{C}$ on $13: 11$ light/dark cycles. Embryonic life staging, aquarium and animal maintenance procedures, diet, and husbandry approaches were completed as described in 'The Zebrafish Book' (Westerfield, 1995). Age of embryo was scored as hours post fertilization (hpf) or days post fertilization (dpf). Embryos were raised at $28.5^{\circ} \mathrm{C}$ using tank water supplemented with $0.01 \%(w / v)$ methylene blue (Sigma Aldrich) from 0 - 2 dpf.

2.2 Microinjection: Microinjection procedures were executed as described previously (Liu et al., 2012). Fertilized embryos were serially collected, cleaned, and segregated 
144 from adults after spawning ( $<0.25 \mathrm{hpf})$. Embryos were mounted on $1.5 \%$ agarose

145 injection plates supplemented with $0.01 \%(\mathrm{w} / \mathrm{v})$ methylene blue (Sigma Aldrich). $2 \mathrm{~nL}$ of $1460.4 \mathrm{mM}$ lepa antisense morpholino oligonucleotides (5'-TTG AGC GGA GAG CTG GAA 147 AAC GCA T -3'), reconstituted in Danieau buffer ([58 mM NaCl, $0.7 \mathrm{mM} \mathrm{KCl}, 0.4 \mathrm{mM}$ $\mathrm{MgSO}_{4}, 0.6 \mathrm{mM} \mathrm{Ca}\left(\mathrm{NO}_{3}\right)_{2}, 5.0 \mathrm{mM}$ HEPES pH 7.6]), were delivered into 1-2 cell stage zebrafish embryos using a IM300 pneumatic microinjector (Narishige). Leptin-a "rescue" injections were prepared with $30 \mu \mathrm{M}$ recombinant zebrafish Leptin-a protein stock solution (50 mM Tris, pH 8.0, > 90\% pure; GenScript) mixed 1:1 with $0.8 \mathrm{mM}$ lepa morpholinos (Gene Tools), final concentration $15 \mu \mathrm{M}$ recombinant zebrafish leptin, 0.4 $\mathrm{mM}$ morpholino, delivered in $2 \mathrm{~nL}$. Injection needles were prepared from $0.58 \mathrm{~mm}$ borosilicate glass capillaries using a P-30 micropipette puller (Stoelting).

2.3 RNA Isolation and Microarray Processing: At $72 \mathrm{hpf}, 5$ dechorionated embryos were pooled together for each biological replicate. This was repeated $8 x$ for uninjected control embryos ( 8 pools of RNA ${ }^{*} 5$ embryos each $=40$ total embryos), $4 x$ for morpholino injected embryos ( 4 pools ${ }^{*} 5$ embryos each $=20$ embryos) and $4 x$ for morpholino + recombinant leptin-injected embryos (4 pools* 5 embryos each=20 embryos). Thus our sample size for microarray analysis was 8 control, 4 lepa morphants, and 4 lepa rescue. RNA was isolated with TRIzol reagent (Thermo Fisher Scientific), DNase treated with the Turbo-DNA-free kit (Ambion), and washed using the RNeasy MinElute cleanup kit (Qiagen). RNA integrity (RIN) was scored with an Agilent 2100 Electrophoretic Bioanalyzer (Agilent). RNA preps were quantified using a Qubit 2.0 Fluorimeter (Thermo Fisher Scientific). Biotinylated cDNA libraries were prepared from equal amounts (250 ng) of high-quality total RNA [RIN $\geq 9.0$ ], [260 : $280=1.9-2.2$ ], [260 : $230=1.9$ - 2.2] using the WT expression kit (Ambion) and the GeneChip terminal labeling kit (Ambion). After fragmentation, cDNA libraries (5.5 ug) were hybridized to single-channel Affymetrix Zebrafish 1.1 ST whole-transcriptome gene array strips (Affymetrix) for 20 hours at $48^{\circ} \mathrm{C}$. Library preparation and microarray scanning procedures were performed by the University of Michigan's Microarray Core Facility

172 following manufacturer guidelines provided for the GeneAtlas system v1.0.4.267

\section{3 (Affymetrix).}


174

175

176

177

178

179

180

181

182

183

184

185

186

187

188

189

190

191

192

193

194

195

196

197

198

199

200

201

202

203

204

2.4 Microarray Statistical Analysis: Microarray .CEL files are deposited in ArrayExpress under accession ID E-MTAB-6548. A total of 16.CEL files, containing probe intensities from each single-channel microarray, were placed into ' $R$ ' statistical environment v3.5.2 (Team, 2017) fitted with Bioconductor v3.4 (Gentleman et al., 2004), and associated library packages. Probeset intensities were derived from .CEL files using the Robust Multichip Average (RMA) method and 'core' summarization in 'oligo' v1.44.0 (Bolstad et al., 2003; Carvalho and Irizarry, 2010; Irizarry et al., 2003a, 2003b). Affymetrix probeset ID's were mapped to annotations from the Zv9 reference assembly with 'pd.zebgene.1.1.st' v3.12.0 (Carvalho, 2015), 'affycoretools' v1.52.2 (MacDonald, 2008), and 'org.Dr.eg.db' v3.6.0 (Carlson, 2017). Prior to hypothesis testing, probesets without gene symbol identifiers were filtered from the dataset. The remaining probesets were compiled to represent expression levels for single genes where each unique gene symbol identifier is represented by one probeset ranked by highest average expression across the series of arrays. Linear modeling for microarray analysis was performed on $\log _{2}$ probeset intensities with 'limma' v3.36.3 (Ritchie et al., 2015) followed by moderation of standard error using the empirical Bayesian method (Smyth, 2005). Three pairwise comparisons were evaluated for reliable differences in digital gene expression measures: lepa morphants compared to uninjected controls; lepa rescue compared to lepa morphant; lepa rescue compared to uninjected controls. Differentially expressed gene (DEG) selection criterions are adjusted p.value $<0.01$ and $\log _{2}$ fold change $<-0.5$ or $>0.5$ because p.value and fold change have collective merit in microarray transcriptomics (Dalman et al., 2012). Differentially expressed Entrez gene identifiers were mapped to zebrafish KEGG pathways $(P<0.05)$ and Gene Ontology databases $(P<0.01)$ using 'clusterProfiler' v3.8.1 (Yu et al., 2012) for each separate pairwise comparison. The method of Benjamini and Hochberg was used to adjust for multiple testing (Benjamini and Hochberg, 1995). KEGG pathway diagrams were rendered by 'pathview' v1.20.0 (Luo and Brouwer, 2013).

2.5 qPCR validation of microarray results. Relative expression for 96 transcripts via qPCR was estimated in the lepa morphant, lepa rescue, and control groups (prepared as detailed in section 2.2) using $\mathrm{RT}^{2}$ Signal Transduction Pathway Finder qPCR Arrays (Qiagen). Each qPCR array was prepared in duplicate ( $n=2$ for control, morphant, and 
205 rescue treatments) with RNA derived from 5 embryos, and equal amounts of total RNA 206 was reverse transcribed with a qScript Flex cDNA synthesis kit (Quanta Bio) with oligo 207 dT primers (along with no template, no primer, and no reverse transcriptase controls).

208 Primer sequences for the qPCR assays are available from the manufacturer (cat\# 209 PAZF-014Z) including 5 reference genes (acta 1b, b2m, hprt1, nono, rp/13a) to which 210 the data were normalized. Assays were run with $\mathrm{RT}^{2}$ SYBR Green Master Mix (Qiagen)

211 on an Applied Biosciences 7300 cycler. Data were analyzed with the Qiagen Gene 212 Globe data analysis web portal (Qiagen). Within the 96 transcripts, those identified as DEG by the microarray analyses were compared across assays (qPCR vs. microarray).

214

215

216

217

218

219

220

221

222

223

224

225

226

227

228

229

230

231

232

233

234

\section{Results}

3.1 Differentially Expressed Genes (DEG's): We previously validated lepa morpholino knockdown using an immunoblot and mismatch morpholinos (Liu et al., 2012). At 72 hpf, lepa morphants have reduced metabolic rate (Dalman et al., 2013), physical malformations pertaining to neurosensory organs (small eyes, otoliths, hindbrain), enlarged pericardial cavity and yolk sac, irregular curvature of the notochord and tail, thinning of spinal nerves, and reduced pigmentation. Injection of both rxLeptin-a and lepa morpholinos alleviates the lepa knockdown phenotype where embryos injected with higher concentrations of rxLeptin-a more closely resemble uninjected control morphology (Liu et al., 2012). Here, microarray expression estimates from 75,212 probesets ( 1.2 million probes) were filtered to represent 26,046 unique gene symbol identifiers derived from the Zv9 reference assembly. In total, $n=16$ microarrays were used to evaluate gene expression data derived from 40 uninjected control embryos, 20 embryos with lepa morpholino knockdown, and 20 embryos with lepa rescue; each microarray sample was derived from a pool of 5 embryos at $72 \mathrm{hpf}$.

Differentially expressed genes (DEG's) have $\log _{2}$ fold change $<-0.5$ or $>0.5$ and adjusted p.value $<0.01$ [Figure 1]. DEG's were rank-ordered by adjusted p.value. Genes that are differentially expressed between lepa morphant and uninjected control microarray samples represent changes in gene expression that correspond to decreased Leptin-a signaling. Similarly, DEG's in the lepa rescue samples compared to uninjected controls present the combinatorial effect of reduced lepa translation (via 
235 morpholino) and supplementation by recombinant protein. Genes that are differentially

236 expressed in lepa rescue compared to lepa morphant samples respond to the induction

237 of Leptin-a signaling (alone) because both conditions were treated with the same

238 concentration of lepa morpholinos. While these microarray data alone cannot

239 distinguish between first or second order gene targets of the zebrafish Leptin-a

240 signaling, this study is the first to measure the whole transcriptome response to reduced

241 leptin signaling in a non-mammal.

242 A total of 19,987 genes were not differentially expressed in any part of the microarray 243 dataset (76.73\%). 1,461 genes were differentially expressed between lepa morphant 244 and control treatments; 425 of the 1,461 probesets were unique to this comparison.

245 Similarly, there were 5,105 DEG's in the lepa rescue treatment compared to the 246 controls; 3,125 of the 5,105 probesets were unique to this comparison. Additionally,

$247 \quad 1,716$ genes were differentially expressed in lepa rescue samples compared to lepa

248 morphants where 329 of the 1,716 probesets were unique to this comparison.

249 Ultimately, 43 genes were differentially expressed among all three pairwise

250 comparisons [Figure 1]. DEG's in each comparison are appended in the

251 Supplementary Gene List. Microinjections, library preparation, and microarray scanning

252 for $n=16$ samples were executed across 5 independent trials. The sample median

253 probeset intensities are consistent across the series of microarrays regardless of scan

254 date or treatment [Supplementary Figure 1]. Principle component analysis illustrates

255 that the cumulative proportion of variance for principle components 1,2 , and 3 is

$25665.97 \%$, or $38.77 \%, 18.58 \%$, and $8.62 \%$, respectively. lepa morphant, lepa rescue, and

257 uninjected control samples form segregated clusters that do not contain members from

258 different treatments, demonstrating that the most variable features in the dataset are

259 more similar among microarrays with a common treatment as opposed to common scan

260 date [Supplementary Figure 1].

261 The 50 top-ranked DEG's from each pairwise comparison are listed in Tables $1-3$.

262 KEGG and GO enrichment analyses were performed on each differentially expressed

263 gene set (separately) to generate inferences on the regulation of the transcriptome by

264 reduced versus induced Leptin-a signaling using knockdown and rescue treatments,

265 respectively. Opsin 1 medium-wave-sensitive 1 (opn1mw1) has the largest reduction in 
266 expression of all genes in both lepa morphant (-6.10 $\log _{2}$ fold change) and lepa rescue 267 (-3.82 $\log _{2}$ fold change) treatments compared to control. In the lepa morphant and

268

269

270

271

272

273

274

275

276

277

278

279

280

281

282

283

284

285

286

287

288

289

290

291

292

293

294

295

296

control contrast, the top- 5 ranked genes include immunoglobulin superfamily DCC subclass member 3-like (LOC100537029, $3.27 \log _{2}$ fold change) and immunoglobin superfamily member $21 \mathrm{~b}$ (igsf21b, $-2.25 \log _{2}$ fold change). Von Willebrand factor ( $v w f$; $3.55 \log _{2}$ fold change) and SI:DKEY-24I24.3 (3.52 $\log _{2}$ fold change) are the two highestranked genes in the lepa rescue treatment compared to the controls. In lepa rescue zebrafish, leptin-b (lepb) is upregulated ( $1.76 \log _{2}$ fold change) compared to controls. In lepa morphant zebrafish, lepa and lepr are differentially expressed (1.01 and $0.77 \log _{2}$ fold changes, respectively) compared to controls which may be compensatory to lepa morphant.

3.2 KEGG Pathway Enrichment: To avoid the inherent problems of multiple comparisons with transcriptomics studies, data reducing techniques that identify classes of genes and pathways that are differentially expressed are common and useful ways of analyzing these datasets. Gene Ontologies (GO) are classifiers used to group gene sets by similarity in function based on phylogenetic, computational, and experimental inferences. GO categories are segregated into biological process (BP), cellular component (CC), and molecular function (MF) components. Similarly, Kyoto Encyclopedia of Genes and Genomes (KEGG) pathway analysis is another classifier that maps interactions within a gene set to coordinated biochemical signaling pathways and molecular functions. KEGG pathway results $(P<0.05)$ are summarized in Table 4 for all three pairwise comparisons; DEG's that map to each enriched KEGG pathway and GO category are appended in the Supplementary Gene List.

Notch signaling is the only differentially expressed KEGG pathway in lepa rescue zebrafish compared to lepa morphants (represented by DEG's dlb, notch1a, notch1b, ep300a, dld, notch3, jag1a, dlc, crebbpb, dv/3a, dla, kat2a) [Table 4]. The highestranked KEGG pathway in lepa morphants compared to control zebrafish is phototransduction (rho, gnat2, rgs9a, grk7a, grk1b, rcvrna, gnat1, saga, pde6a, pde6b, calm1a, calm1b, guca1d, grk1a), followed by ribosome biogenesis in eukaryotes ( $n m d 3$, tb/3, rexo1, rpp25l, mphosph10, rcl1, gar1, utp18, pwp2h, dkc1, wdr3, mdn1), notch signaling (dlb, notch1a, dld, dla, notch1b, notch3, jag1a, her15.1, dlc, rbpjl), then 
297 neuroactive ligand-receptor interaction (grin1b, gabrd, grm1a, grin2ab, drd4b, gria3a,

298 grm3, grin1a, gabrb2, gabbr2, chrna6, lepa, si:dkey-1h24.2, oxtr, thrab,

299

300

301

302

303

304

305

306

307

308

309

310

311

312

313

314

315

316

317

318

319

320

321

322

323

324

325

326

327

LOC100330554, grik1a, crhr1, drd1b, vipr2, chrm4a, calcr, oprm1, grm6b, LOC562831, vipr1b, lepr, gria1b, p2rx2, LOC100334689, gria2b, glra3, grm6a, gnrhr4). lepa rescue compared to control samples identify neuroactive ligand-receptor interaction (94 DEG's; Figure 2) and phototransduction (gnat2, rho, grk7a, saga, gnat1, grk1a, rcvrna, rgs9a, calm1a, calm1b, exorh, grk1b, rgs9b) similar to lepa morphants. However, rescue vs. control also identifies phosphatidylinositol signaling (Figure 3; mtmr1a, ip6k1, LOC567728, itpr2, mtmr7b, inpp5e, mtmr3, plcd3a, plcb4, pip5k1ca, ipmkb, itpr1a, pi4kaa, calm1a, dgkh, LOC100333801, dgkza, plcg2, pik3r1, prkcba, calm1b, ppip5k2, synj1, prkcbb, inpp5f, mtmr6, plcg1, dgke, itpkca) and glycosaminoglycan biosynthesis (xylt1, ext1c, ndst3, hs3st1/1, hs6st2, glcea, hs3st2, hs6st1a, ext/3, glceb, hs3st3b1b).

The top-50 DEG's in lepa morphants compared to controls are clustered to illustrate expression patterns among genes and microarray samples [Figure 4a]. All microarray samples clustered with members from the same treatment. The heatmap includes 5 transcription factors (neurog1, pou2f2a, insm1a, brf1a, foxn4) as well as components of the Delta/Notch and phototransduction genes (demarcated by orange and purple, respectively). Phototransduction and delta/notch genes display reciprocal trends in expression with respect to treatment (down with morphants, up with rescue) indicating that they may be coregulated. Delta/Notch has high expression in lepa morphants and low expression in both the control and lepa rescue samples. Conversely, phototransduction genes have high expression in controls but low expression in lepa morphant and rescue treatments. Figure $4 \mathrm{~b}$ clusters homologous gene targets that are regulated by the mammalian leptin signaling pathway (lepa, lepb, lepr, LOC794738, LOC100537326, akt3, pik3r1, prkaa2, socs2, socs5b, socs9, npy8br, npy8ar, mc5ra, foxo1b, jak2a, jak2b, camkk2, map3k12, agrp2, cart1). These targets are as identified in mammals by Park and Ahima (2014). Figure 4B also documents genes displaying large fold changes ( $v w f$, opn1/w1, igsf21b, LOC100537029), and others that regulate lipid (acacb, dagla, daglb, Ipl, crot, plin1, cpt1b, pank2) or RNA metabolism (smg5, dicer1, LOC796505, LOC570775).

3.3 Gene Ontology Enrichment: The lepa morphant compared to control GO data were 
328 generated from 1,461 DEG's (970 Entrez gene ID's); lepa morphants have 54 enriched

329 biological process terms $(P<0.01)$ including visual perception, ncRNA metabolic

330 process, rRNA metabolic process, ribosomal large/small subunit biosynthesis,

331

332

333

334

335

336

337

338

339

340

341

342

343

344

345

346

347

348

349

350

351

352

353

354

355

356

357

358 neurological system process, protein-chromophore linkage, spinal cord development, nervous system development and neuron differentiation, regulation of neurotransmitter levels and secretion, synaptic signaling, and regulation of neurogenesis [Figure 5]. The GO data for the lepa rescue and morphant contrast were generated from 1,716 DEG's (999 Entrez gene ID's) that correspond to 22 enriched terms including sensory perception, Notch signaling, brain/head/gland/ventral spinal cord development, regionalization, olfactory and semaphorin receptor activity, and protein tyrosine kinase activity [Figure 6]. Biological process Gene Ontologies from the lepa rescue and control comparison were produced using 5,105 DEG's (3,036 Entrez gene ID's); lepa rescue has 39 enriched biological process terms including JAK-STAT cascade, nervous system process, synaptic signaling, synapse organization, sensory perception, regulation of voltage-gated calcium channels, neurotransmitter secretion, and phototransduction [Figure 7]. Genes that map to each $\mathrm{GO}$ category in Figures $5-7$ are appended in the Supplementary Gene List.

From 1,461 DEG's in lepa morphants compared to controls, there were 112 transcription factors (7.67\%) [Supplementary Figure 2]. There are 5 differentially expressed transcription factors with a $\log _{2}$ fold change greater than 2 (foxn 4 , fos/1a, $c b x 7 a$, and atf5a). Two transcription factors (pou2f2a, insm1a) ranked in the top-10 of all 1,461 DEG's between lepa morphants and controls [Table 1]. Additionally, the Gene Ontology enrichment analysis for transcription factor biological process has 65 terms that envelop many aspects of development (spinal cord, endocrine system, pancreas, brain, head, neural retina, nervous system, epithelium), as well as regulation of neurogenesis, neuron differentiation, cell fate commitment, and hindbrain morphogenesis [Supplementary Figure 3].

3.4 qPCR validation of the microarray: Several genes within the $\mathrm{QPCR}$ microarray were also identified as DEG in the microarray experiments (10 for morphant vs. control and 5 for rescue vs. control; Supplementary Figure 4). Fold differences $>1$ are positive changes in expression, and $<1$ are negative. The direction of expression change is the 
359

360

361

362

363

364

365

366

367

368

369

370

371

372

373

374

375

376

377

378

379

380

381

382

383

384

385

386

387

388

same between microarray and qPCR experiments for 8 out of 10 genes for the comparison of morphants to control. For the rescue vs. control comparison, 1 out of 5 genes has the same direction of change when expression is measured by microarray vs. qPCR.

\section{Discussion}

Null mutations in leptin (lep) or leptin receptor (lepr) impair lipid metabolism, endocrine signaling, and energy homeostasis in humans and rodents, but there are few nonmammal model organisms with reduced leptin signaling. Mammalian leptin is widely characterized in adults, and to a lesser extent in juveniles or embryos (Antczak and Van Blerkom, 1997). Embryonic leptin expression precludes terminal adipocyte differentiation in mammals suggesting that leptin modulates embryogenesis apart from its role as an adipocytokine (Antczak and Van Blerkom, 1997). Given the intrinsic limitations of in vivo embryonic leptin manipulation using mammals, we tested transcriptomic response to leptin manipulation in developing zebrafish using lepa morpholino morphants. Our findings highlight roles for Leptin-a tied to the regulation of central endocrine and phosphatidylinositol signaling gene expression in early zebrafish development which align with canonical targets of leptin signal transduction cascades in mammals.

Zebrafish lepa and lepb are co-expressed in zebrafish pituitary which may mediate endocrine and local (paracrine, autocrine) axes, respectively (Gorissen et al., 2009). Affirming a role for Leptin-a signaling in the central nervous system, neuroactive ligandreceptor interactions are enriched KEGG pathways in lepa morphant and rescue zebrafish compared to controls, but fewer genes respond to morphant as opposed to rescue suggesting there are differential responses to reduced versus induced Leptin-a signaling [Figure 2]. Additionally, lepa rescue DEG's are analogous to neuroendocrine factors associated with the human leptin pathway (and classical mediators of changes in appetite and metabolism), including agouti-related peptide-2 (agrp2), cocaine-andamphetamine-regulated transcript 1 (cart1), neuropeptide $\mathrm{Y}$ receptor Y8 (npy8ar, npy $8 b r)$, and melanocortin receptor $5 \mathrm{a}(\mathrm{mc} 5 \mathrm{ra}$ ) [Figure $4 \mathrm{~b}$ ]. Zebrafish homologs of the human lep/pi3k/akt, and lep/shp2/mapk cascades are differentially regulated in lepa 
389 rescue (but not lepa morphant) treatments including protein kinase B (akt3), 390 phosphatidylinositol 3-kinase (pik3r1), insulin receptor substrate (LOC794738, 391 LOC100537326), Janus kinase 2 (jak2a, jak2b), forkhead box O1 (foxo1b), AMP392 activated protein kinase (prkaa2), acetyl-CoA carboxylase beta (acacb), 393 calcium/calmodulin kinase (camkk2), mitogen-activated protein kinase kinase kinase 12 394 (map3k12), and suppressors of cytokine signaling (socs2, socs5b, socs9) [Figure 4b].

395 Consequently, KEGG phosphatidylinositol signaling [Figure 3] and GO biological process JAK/STAT cascade [Figure 7] are enriched in lepa rescue zebrafish. In addition to prkaa2 and acacb, lipolytic factors also respond to lepa rescue (but not morphant) including pantothenate kinase 2 (pank2) which mediates a critical step of coenzyme-A biosynthesis, lipoprotein lipase ( $/ p /)$, diacylglycerol lipase (dagla, daglb), perilipin-1 (plin1), carnitine O-octanoyltransferase (crot), and muscle carnitine palmitoyltransferase 1B (cpt1b) [Figure 4b].

Although lists of differentially expressed genes are a useful outcome of these studies, perhaps the most relevant results come from GO (gene ontology) and pathway analyses (e.g. KEGG). The goal of these analyses is to uncover functions/pathways that are statistically over/underrepresented in the treatment. Once a pathway is identified, all genes within the pathway are legitimate targets for downstream hypothesis testing, regardless of whether each gene in the pathway is differentially expressed (Kahtri et al. 2012). Notch signaling is differentially regulated between lepa morphant and rescue treatments [Figure 4a; Table 4]. Notch signaling is a conserved juxtacrine signaling pathway that, in addition to many other roles, directs neuron cell fate (Wakeham et al., 1997). Notch signaling, neurogenesis, and neural differentiation respond to lepa knockdown [Figure 4a; Supplementary Figure 3]. GO enrichment pertaining to synaptic signaling, sensory perception, and neurotransmitter secretion is enriched in both lepa morphant and rescue treatments compared to controls [Figures 5 and 7]. ob/ob and $d b / d b$ rodents have impaired nerve fiber extensions in arcuate nucleus (Bouret et al., 2004). Similarly, lepa and lepr zebrafish morphants have thinning of spinal nerves along with malformations of sensory organs (Liu et al., 2012) indicating that there may be conserved roles between the embryonic zebrafish and human leptin signaling pathways tied to neurogenesis and synapse organization. This 
420 pathway analysis may also be less susceptible to gene:gene variation among replicates.

421 Validation of expression data for 10 genes between the microarray and control groups is 422 generally good, with 8/10 genes matching in the direction of change (e.g. up or down 423 both in microarray and qPCR data, Supplementary Figure 4). However, agreement 424 between the two methods for the rescue vs. control comparison is poor (1 of 5). We 425 speculate that this treatment is inherently more complex (both knockdown with $\mathrm{MO}$ and rescue with $\mathrm{RX}$ leptin), and thus the response more variable. Other transcriptomics studies also report relatively low rates of agreement between transcriptomic and qPCR data (Cui et al., 2014).

Other laboratories investigated transcriptomic response to leptin manipulation in nonmammals. Denver's laboratory measured individual gene response and transcriptome response to leptin incubation in Xenopus brain preparations (Cui et al., 2014). They noted strong response of socs genes, similar to this study (Figure 4b). Recently, Borski's laboratory published results of an ex vivo transcriptomic study on adult tilapia pituitary tissue treated with tilapia Leptin-a in culture (Douros et al., 2018). The overwhelming effect was increased carbohydrate metabolism, consistent with a leptin knockout in zebrafish (Michel et al. 2016). Several aspects of our study are consistent with Douros et al. (2018), such as protein kinase B (akt3), Janus kinase 2 (jak2a, jak2b), insulin receptor substrate (LOC794738, LOC100537326), AMP-activated protein kinase (prkaa2), acetyl-CoA carboxylase beta (acacb) [Figure 4b], and the general stimulatory effects on ribosome assembly and function [Table 4]. Differences between the two studies likely reflect developmental window (embryos vs. adults), and tissue (whole embryo vs pituitary and liver); it is not surprising that leptin may have different effects on the transcriptome of developing whole embryos versus specific tissues of adults, even in the same species.

Phototransduction genes elicit the same response to lepa morphant and rescue treatments compared to controls suggesting this effect is not directly correlated with Leptin-a signaling [Figure 4a; Table 4]. Correlations between the lepa morphant dataset and unrelated morpholinos suggest that there may be a phototransduction artifact of morpholinos which is often manifested as reduced eye size and opsin expression. 
451 are typically seen at much higher morpholino dose/embryo than used in this study.

452 Although we assert that our results generally reflect the effects of lepa knockdown in

453 zebrafish embryos, it is also likely that the general effects of morpholino

454 oligonucleotides are reflected in the data. Eukaryotic gene expression is regulated at

455 multiple levels including transcriptional machinery and epigenetic factors, mRNA

456 processing and transport, and post-translational modifications. The transcriptome

457 represents the complete spectrum of RNA species that are present at one reference

458 point in time. Expression microarrays are molecular technologies adapted to

qualitatively and semi-quantitatively describe transcribed regions of the genome using a single experiment. Structurally, antisense morpholino oligonucleotides (MO) are short 25 mer, nucleic acid analogues with alternative morpholine rings in place of the native deoxyribose and ribose moieties present in DNA and RNA, respectively (Summerton and Weller, 1997). Uncharged phosphodiamorate linkages between morpholino nucleotides juxtapose the negatively charged phosphodiester backbones of native DNA/RNA. These structural rearrangements confer resistance to RNase $\mathrm{H}$ nucleolytic cleavage while the uncharged MO backbone reduces off-target electrostatic interactions during delivery and diffusion (Summerton, 1999). MO's are generally directed against the translation initiation site of a sense strand mRNA as described here. Alternatively, mRNA transport, maturation, and processing can be manipulated by directing MO's against splice boundaries of precursor mRNA's (Kloosterman et al., 2007). Gene knockdown is catalyzed by complementary base-pairing between antisense $\mathrm{MO}$ and target mRNA. Translation-blocking MO:mRNA hybrids sterically exclude the ribosome from executing translation which leads to reduced target protein synthesis and/or impaired processing of target RNA (Bill et al., 2009; Summerton, 2007). However, MO's generally have a temporal range of effectiveness. Cytological concentrations of MO's are reduced with every subsequent cell division which restricts most MO applications to early life stages (Nasevicius and Ekker, 2000).

Morpholinos have mistargeting potentials associated with tp53 expression programs, neuron death, and hindbrain development; p53 morpholino co-morphants alleviate most of these artifacts (Gerety and Wilkinson, 2011; Robu et al., 2007). Zebrafish lepa morphants share morphological features with zebrafish morphants from other target 
482 genes, such as pericardial edema, enlarged yolk sac, reduced eye and otolith size, as 483 well as tail curvature (Bagci et al., 2015; Kok et al., 2015; Kwon, 2016; Liu et al., 2012; 484 Pham et al., 2007; Robu et al., 2007). It is plausible that the formation of double

485

486

487

488

489

490

491

492

493

494

495

496

497

498

499

500

501

502

503

504

505

506

507

508

509

510

511

stranded morpholino:RNA hybrids may enhance the RNA-induced silencing complex as ncRNA metabolism and RNA processing are among the top-ranked GO terms in lepa morphant zebrafish [Figure 5] which include Smg-5 nonsense mediated mRNA decay factor (smg5), Dicer-1 (dicer1), protein argonaute-1-like (LOC570775), and putative ATP-dependent RNA helicase DHX33-like (LOC796505) [Figure 4b].

Finally, we understand that the interaction among the morpholino and recombinant protein is/are likely complex. The degree to which the morphants have reduced expression, and the degree to which the recombinant leptin replaces what is reduced by morpholinos is unlikely a perfect match. This undoubtedly contributes to different pathways identified in each of the comparisons.

\section{Conclusions}

These microarray data describe cell signaling pathways and gene targets regulated by embryonic zebrafish Leptin-a. Differentially expressed genes from the lepa rescue zebrafish embryos influence expression of genes that participate in central endocrine and phosphatidylinositol signaling pathways which agrees with the function of leptin signaling in mammals. Regulators of metabolism respond to lepa rescue that agree with lep/ampk/acc and lep/pi3k/akt cascades in human (Minokoshi et al., 2002) [Figure 4B]. There are also markers consistent with increased central leptin signaling in the CNS among lepa rescue zebrafish which includes cocaine-and-amphetamine regulated transcript like-1 (cart1; 0.99 log2 fold change) and agouti-related peptide-2 (agrp2; -1.21 log2 fold change). Although we did not identify differentially expressed members of the STAT transcription factor family, Janus kinase 2 (jak2a, jak2b), insulin receptor substrate (LOC794738, LOC100537326), insulin receptor a (insra), Ser/Thr protein kinase B (akt3), PI3K (pik3r1), and forkhead box 01 (foxo1b) are differentially expressed in lepa rescue zebrafish which aligns with coregulation of the central $\mathrm{PI3K} / \mathrm{Akt} / \mathrm{FoxO} 1$ cascade by leptin and insulin receptor signaling in mammals (Xu et al., 2005). Taken together, our results propose that cell signaling pathways regulated by 
512 the A-type zebrafish leptin paralog align with human and rodent leptins including

513 lepA/pi3k/akt and lepa/ampk/acc but functional assays using fish leptin signaling

514 knockouts are needed to validate these gene expression data.

515

516

\section{References}

517

518

Antczak, M., and Van Blerkom, J. (1997). Oocyte influences on early development: the regulatory proteins leptin and STAT3 are polarized in mouse and human oocytes and differentially distributed within the cells of the preimplantation stage embryo. Mol. Hum. Reprod. 3, 1067-1086.

Bagci, E., Heijlen, M., Vergauwen, L., Hagenaars, A., Houbrechts, A.M., Esguerra, C.V., Blust, R., Darras, V.M., and Knapen, D. (2015). Deiodinase knockdown during early zebrafish development affects growth, development, energy metabolism, motility and phototransduction. PloS One 10, e0123285.

Baskin, D.G., Schwartz, M.W., Seeley, R.J., Woods, S.C., Porte Jr, D., Breininger, J.F., Jonak, Z., Schaefer, J., Krouse, M., and Burghardt, C. (1999). Leptin receptor long-form splice-variant protein expression in neuron cell bodies of the brain and co-localization with neuropeptide Y mRNA in the arcuate nucleus. J. Histochem. Cytochem. 47, 353362.

Benjamini, Y., and Hochberg, Y. (1995). Controlling the false discovery rate: a practical and powerful approach to multiple testing. J. R. Stat. Soc. Ser. B Methodol. 289-300.

Bieber, L.L., Abraham, T., and Helmrath, T. (1972). A rapid spectrophotometric assay

Bill, B.R., Petzold, A.M., Clark, K.J., Schimmenti, L.A., and Ekker, S.C. (2009). A primer for morpholino use in zebrafish. Zebrafish 6, 69-77.

Bolstad, B.M., Irizarry, R.A., Åstrand, M., and Speed, T.P. (2003). A comparison of normalization methods for high density oligonucleotide array data based on variance

Bouret, S.G., Draper, S.J., and Simerly, R.B. (2004). Trophic action of leptin on hypothalamic neurons that regulate feeding. Science $304,108-110$.

Bradford, M.M. (1976). A rapid and sensitive method for the quantitation of microgram 254.

Carlson, M. (2017). org.Dr.eg.db: Genome wide annotation for Zebrafish. R Package Version 3.4.1. 
549 Carvalho, B.S. (2015). pd.zebgene.1.1.st: Platform Design Info for Affymetrix ZebGene550 1_1-st. R Package Version 3.12.0.

551 Carvalho, B.S., and Irizarry, R.A. (2010). A framework for oligonucleotide microarray 552 preprocessing. Bioinformatics 26, 2363-2367.

553 Chen, H., Charlat, O., Tartaglia, L.A., Woolf, E.A., Weng, X., Ellis, S.J., Lakey, N.D., 554 Culpepper, J., More, K.J., and Breitbart, R.E. (1996). Evidence that the diabetes gene 555 encodes the leptin receptor: identification of a mutation in the leptin receptor gene in $556 d b / d b$ mice. Cell 84, 491-495.

557 Chisada, S., Kurokawa, T., Murashita, K., Rønnestad, I., Taniguchi, Y., Toyoda, A., 558 Sakaki, Y., Takeda, S., and Yoshiura, Y. (2014). Leptin receptor-deficient (knockout) 559 medaka, Oryzias latipes, show chronical up-regulated levels of orexigenic 560 neuropeptides, elevated food intake and stage specific effects on growth and fat 561 allocation. Gen. Comp. Endocrinol. 195, 9-20.

Chu, D.L.H., Li, V.W.T., and Yu, R.M.K. (2010). Leptin: clue to poor appetite in oxygenstarved fish. Mol. Cell. Endocrinol. 319, 143-146.

564

565

566

567

568

569

570

571

572

573

574

575

576

577

578

579

580

581

582

583

584

585
Cohen, P., Zhao, C., Cai, X., Montez, J.M., Rohani, S.C., Feinstein, P., Mombaerts, P., and Friedman, J.M. (2001). Selective deletion of leptin receptor in neurons leads to obesity. J. Clin. Invest. 108, 1113-1121.

Coleman, D.L. (1973). Effects of parabiosis of obese with diabetes and normal mice. Diabetologia 9, 294-298.

Cui M.Y., Hu C.K., Pelletier C., Dziuba A., Slupski R.H., Li C., Denver R.J. (2014). Ancient origins and evolutionary conservation of intracellular and neural signaling pathways engaged by the leptin receptor. Endocrinology. 155(11):4202-14.

Dalman, M.R., Deeter, A., Nimishakavi, G., and Duan, Z.-H. (2012). Fold change and pvalue cutoffs significantly alter microarray interpretations. In BMC Bioinformatics, (BioMed Central), p. S11.

Dalman, M.R., Liu, Q., King, M.D., Bagatto, B., and Londraville, R.L. (2013). Leptin expression affects metabolic rate in zebrafish embryos (D. rerio). Front. Physiol. 4:160.

Douros, J.D., Baltzegar, D.A., Reading, B.J., Seale, A.P., Lerner, D.T., Grau, E.G.G., and Borski, R.J. (2018). Leptin stimulates cellular glycolysis through a STAT3 dependent mechanism in Tilapia. Front. Endocrinol. 9:465.

Dunn SL ${ }^{1}$, Björnholm M, Bates SH, Chen Z, Seifert M, Myers MG Jr.(2005). Feedback inhibition of leptin receptor/Jak2 signaling via Tyr1138 of the leptin receptor and suppressor of cytokine signaling 3. Mol. Endocrinol. 19: 925-938.

Farooqi, I.S., Jebb, S.A., Langmack, G., Lawrence, E., Cheetham, C.H., Prentice, A.M., 
586 Hughes, I.A., McCamish, M.A., and O'rahilly, S. (1999). Effects of recombinant leptin 587 therapy in a child with congenital leptin deficiency. N. Engl. J. Med. 341, 879-884.

588 Farooqi, I.S., Matarese, G., Lord, G.M., Keogh, J.M., Lawrence, E., Agwu, C., Sanna, 589 V., Jebb, S.A., Perna, F., and Fontana, S. (2002). Beneficial effects of leptin on obesity, 590 T cell hyporesponsiveness, and neuroendocrine/metabolic dysfunction of human 591 congenital leptin deficiency. J. Clin. Invest. 110, 1093-1103.

592 Farooqi, I.S., Wangensteen, T., Collins, S., Kimber, W., Matarese, G., Keogh, J.M., 593 Lank, E., Bottomley, B., Lopez-Fernandez, J., and Ferraz-Amaro, I. (2007). Clinical and 594 molecular genetic spectrum of congenital deficiency of the leptin receptor. N. Engl. J. 595 Med. 356, 237-247.

596 597

598

599

600

601

602

603

604

605

606

607

608

609

610

611

612

613

614

615

616

617

618

619

620

621

622

623

624

Friedman, J.M., and Halaas, J.L. (1998). Leptin and the regulation of body weight in mammals. Nature 395, 763-770.

Gentleman, R.C., Carey, V.J., Bates, D.M., Bolstad, B., Dettling, M., Dudoit, S., Ellis, B., Gautier, L., Ge, Y., and Gentry, J. (2004). Bioconductor: open software development for computational biology and bioinformatics. Genome Biol. 5, R80.

Gerety, S.S., and Wilkinson, D.G. (2011). Morpholino artifacts provide pitfalls and reveal a novel role for pro-apoptotic genes in hindbrain boundary development. Dev. Biol. 350, 279-289.

Ghilardi, N., Ziegler, S., Wiestner, A., Stoffel, R., Heim, M.H., and Skoda, R.C. (1996). Defective STAT signaling by the leptin receptor in diabetic mice. Proc. Natl. Acad. Sci. 93, 6231-6235.

Gibson, W.T., Farooqi, I.S., Moreau, M., DePaoli, A.M., Lawrence, E., O'rahilly, S., and Trussell, R.A. (2004). Congenital leptin deficiency due to homozygosity for the $\Delta 133 G$ mutation: report of another case and evaluation of response to four years of leptin therapy. J. Clin. Endocrinol. Metab. 89, 4821-4826.

Gong N, Jönsson E, Björnsson BT. (2016). Acute anorexigenic action of leptin in rainbow trout is mediated by the hypothalamic Pi3k pathway. J. Mol. Endocrinol. $56(3): 227-38$.

Gorissen, M., Bernier, N.J., Nabuurs, S.B., Flik, G., and Huising, M.O. (2009). Two divergent leptin paralogues in zebrafish (Danio rerio) that originate early in teleostean evolution. J. Endocrinol. 201, 329-339.

Grim, J.M., Miles, D.R.B., and Crockett, E.L. (2010). Temperature acclimation alters oxidative capacities and composition of membrane lipids without influencing activities of enzymatic antioxidants or susceptibility to lipid peroxidation in fish muscle. J. Exp. Biol. 213: $445-452$.

Hansen, C.A., and Sidell, B.D., (1983). Atlantic hagfish cardiac muscle: Metabolic basis of tolerance to anoxia. Am. J. Physiol. 244: R356-R362. 
Howe, K., Clark, M.D., Torroja, C.F., Torrance, J., Berthelot, C., Muffato, M., Collins, J.E., Humphray, S., McLaren, K., and Matthews, L. (2013). The zebrafish reference genome sequence and its relationship to the human genome. Nature 496, 498.

Irizarry, R.A., Hobbs, B., Collin, F., Beazer-Barclay, Y.D., Antonellis, K.J., Scherf, U., and Speed, T.P. (2003a). Exploration, normalization, and summaries of high density oligonucleotide array probe level data. Biostatistics 4, 249-264.

Irizarry, R.A., Bolstad, B.M., Collin, F., Cope, L.M., Hobbs, B., and Speed, T.P. (2003b). Summaries of Affymetrix GeneChip probe level data. Nucleic Acids Res. 31, e15-e15.

Jaillon, O., Aury, J.-M., Brunet, F., Petit, J.-L., Stange-Thomann, N., Mauceli, E., Bouneau, L., Fischer, C., Ozouf-Costaz, C., and Bernot, A. (2004). Genome duplication in the teleost fish Tetraodon nigroviridis reveals the early vertebrate proto-karyotype. Nature 431, 946.

Jo, Y.-H., Chen, Y.-J.J., Chua, S.C., Talmage, D.A., and Role, L.W. (2005). Integration of endocannabinoid and leptin signaling in an appetite-related neural circuit. Neuron 48, 1055-1066.

Kang, J., Hu, J., Karra, R., Dickson, A.L., Tornini, V.A., Nachtrab, G., Gemberling, M., Goldman, J.A., Black, B.L., and Poss, K.D. (2016). Modulation of tissue repair by regeneration enhancer elements. Nature 532, 201.

Khatri, P., Sirota, M., and Butte, A.J. (2012).Ten years of pathway analysis: Current approaches and outstanding challenges. PLoS Comput Biol 8(2): e1002375.

Kloosterman WP1, Lagendijk AK, Ketting RF, Moulton JD, Plasterk RH.(2007). Targeted inhibition of miRNA maturation with morpholinos reveals a role for miR-375 in pancreatic islet development. PLoS Biol. 5(8):e203.

Kok, F.O., Shin, M., Ni, C.-W., Gupta, A., Grosse, A.S., van Impel, A., Kirchmaier, B.C., Peterson-Maduro, J., Kourkoulis, G., and Male, I. (2015). Reverse genetic screening reveals poor correlation between morpholino-induced and mutant phenotypes in zebrafish. Dev. Cell 32, 97-108.

Kurokawa, T., Uji, S., and Suzuki, T. (2005). Identification of cDNA coding for a homologue to mammalian leptin from pufferfish, Takifugu rubripes. Peptides 26, 745750.

Kwon, H.-J. (2016). Vitamin D receptor signaling is required for heart development in zebrafish embryo. Biochem. Biophys. Res. Commun. 470, 575-578.

Lee, G.H., Proenca, R., Montez, J.M., Carroll, K.M., Darvishzadeh, J.G., Lee, J.I., and Friedman, J.M. (1996). Abnormal splicing of the leptin receptor in diabetic mice. Nature 379, 632-635. 
662 Liu, Q., Chen, Y., Copeland, D., Ball, H., Duff, R.J., Rockich, B., and Londraville, R.L. 663 (2010). Expression of leptin receptor gene in developing and adult zebrafish. Gen.

664 Comp. Endocrinol. 166, 346-355.

665

666

667

668

669

670

671

672

673

674

675

676

677

678

679

680

681

682

683

684

685

686

687

688

689

690

691

692

693

694

695

696

Liu, Q., Dalman, M., Chen, Y., Akhter, M., Brahmandam, S., Patel, Y., Lowe, J., Thakkar, M., Gregory, A.-V., and Phelps, D. (2012). Knockdown of leptin A expression dramatically alters zebrafish development. Gen. Comp. Endocrinol. 178, 562-572.

Londraville, R.L., Macotela, Y., Duff, R.J., Easterling, M.R., Liu, Q., and Crespi, E.J. (2014). Comparative endocrinology of leptin: assessing function in a phylogenetic context. Gen. Comp. Endocrinol. 203, 146-157.

Londraville, R.L., Prokop, J.W., Duff, R.J., Liu, Q., and Tuttle, M. (2017). On the molecular evolution of leptin, leptin receptor, and endospanin. Front. Endocrinol. 8, 58.

Luo, W., and Brouwer, C. (2013). Pathview: an R/Bioconductor package for pathwaybased data integration and visualization. Bioinformatics 29, 1830-1831.

MacDonald, J.W. (2008). affycoretools: Functions useful for those doing repetitive analyses with Affymetrix GeneChips. R Package Version 1.

Maffei, M., Halaas, J., Ravussin, E., Pratley, R.E., Lee, G.H., Zhang, Y., Fei, H., Kim, S., Lallone, R., and Ranganathan, S. (1995). Leptin levels in human and rodent: measurement of plasma leptin and ob RNA in obese and weight-reduced subjects. Nat. Med. 1, 1155-1161.

Michel, M., Page-McCaw, P.S., Chen, W., and Cone, R.D. (2016). Leptin signaling regulates glucose homeostasis, but not adipostasis, in the zebrafish. Proc. Natl. Acad. Sci. 113, 3084-3089.

Minokoshi, Y., Kim, Y.-B., Peroni, O.D., Fryer, L.G., Müller, C., Carling, D., and Kahn, B.B. (2002). Leptin stimulates fatty-acid oxidation by activating AMP-activated protein kinase. Nature 415, 339-343.

Montague, C.T., Farooqi, I.S., Whitehead, J.P., Soos, M.A., Rau, H., Wareham, N.J., Sewter, C.P., Digby, J.E., Mohammed, S.N., and Hurst, J.A. (1997). Congenital leptin deficiency is associated with severe early-onset obesity in humans. Nature 387, 903.

Münzberg, H., Flier, J.S., and Bjørbæk, C. (2004). Region-specific leptin resistance within the hypothalamus of diet-induced obese mice. Endocrinology 145, 4880-4889.

Nasevicius, A., and Ekker, S.C. (2000). Effective targeted gene 'knockdown' in zebrafish. Nat. Genet. 26, 216.

Park, H.-K., and Ahima, R.S. (2014). Leptin signaling. F1000prime Rep. 6.

Pelleymounter, M.A., Cullen, M.J., Baker, M.B., Hecht, R., Winters, D., Boone, T., and Collins, F. (1995). Effects of the obese gene product on body weight regulation in ob/ob 
697

698

699

700

701

702

703

704

705

706

707

708

709

710

711

712

713

714

715

716

717

718

719

720

721

722

723

724

725

726

727

728

729

730

731

732

mice. Science $269,540-543$.

Pham, V.N., Lawson, N.D., Mugford, J.W., Dye, L., Castranova, D., Lo, B., and Weinstein, B.M. (2007). Combinatorial function of ETS transcription factors in the developing vasculature. Dev. Biol. 303, 772-783.

Ritchie, M.E., Phipson, B., Wu, D., Hu, Y., Law, C.W., Shi, W., and Smyth, G.K. (2015). limma powers differential expression analyses for RNA-sequencing and microarray studies. Nucleic Acids Res. 43, e47-e47.

Robu, M.E., Larson, J.D., Nasevicius, A., Beiraghi, S., Brenner, C., Farber, S.A., and Ekker, S.C. (2007). p53 activation by knockdown technologies. PLoS Genet. 3, e78.

Savan, R., and Sakai, M. (2006). Genomics of fish cytokines. Comp. Biochem. Physiol. Part D Genomics Proteomics 1, 89-101.

Schwartz, M.W., Seeley, R.J., Woods, S.C., Weigle, D.S., Campfield, L.A., Burn, P., and Baskin, D.G. (1997). Leptin increases hypothalamic pro-opiomelanocortin mRNA expression in the rostral arcuate nucleus. Diabetes 46, 2119-2123.

Smyth, G.K. (2005). Limma: linear models for microarray data. In Bioinformatics and Computational Biology Solutions Using R and Bioconductor, (Springer), pp. 397-420.

Summerton, J. (1999). Morpholino antisense oligomers: the case for an RNase Hindependent structural type. Biochim. Biophys. Acta BBA-Gene Struct. Expr. 1489, 141158.

Summerton, J.E. (2007). Morpholino, siRNA, and S-DNA compared: impact of structure and mechanism of action on off-target effects and sequence specificity. Curr. Top. Med. Chem. 7, 651-660.

Summerton, J., and Weller, D. (1997). Morpholino antisense oligomers: design, preparation, and properties. Antisense Nucleic Acid Drug Dev. 7, 187-195.

Tanida M, Yamamoto N, Morgan DA, Kurata Y, Shibamoto T, Rahmouni K. (2015). Leptin receptor signaling in the hypothalamus regulates hepatic autonomic nerve activity via phosphatidylinositol 3-kinase and AMP-activated protein kinase.J Neurosci. 35(2):474-84.

Team, R.C. (2017). R: a language and environment for statistical computing. Vienna, Austria: R Foundation for Statistical Computing; 2017.

Vaisse, C., Halaas, J.L., Horvath, C.M., Darnell Jr, J.E., Stoffel, M., and Friedman, J.M. (1996). Leptin activation of Stat3 in the hypothalamus of wild-type and ob/ob mice but not $d b / d b$ mice. Nat. Genet. 14, 95.

Wakeham, A., Correia, K.M., Samper, E., Brown, S., Aguilera, R.J., Nakano, T., Honjo, T., Mak, T.W., Rossant, J., and Conlon, R.A. (1997). Conservation of the Notch 
733 signalling pathway in mammalian neurogenesis. Development 124, 1139-1148.

734 Westerfield, M. (1995). The zebrafish book: a guide for the laboratory use of zebrafish 735 (Brachydanio rerio) (University of Oregon press).

736 Wu K, Tan XY, Wei CC, You WJ, Zhuo MQ, Song YF. (2016A). Isolation and

737 expression analysis of STAT members from Synechogobius hasta and their roles

738 in leptin affecting lipid metabolism. Int. J. Mol. Sci. 17(3):406.

739

740 Wu K, Tan XY, Xu YH, Chen QL, Pan YX. (2016B). JAK and STAT members of yellow 741 catfish Pelteobagrus fulvidraco and their roles in leptin affecting lipid metabolism. Gen 742 Comp Endocrinol. 2016 226:14-26.

743

744 Xu, A.W., Kaelin, C.B., Takeda, K., Akira, S., Schwartz, M.W., and Barsh, G.S. (2005).

$745 \mathrm{PI}$ K integrates the action of insulin and leptin on hypothalamic neurons. J. Clin. Invest.

746 115, 951-958.

747 Yu, G., Wang, L.-G., Han, Y., and He, Q.-Y. (2012). clusterProfiler: an R package for 748 comparing biological themes among gene clusters. Omics J. Integr. Biol. 16, 284-287.

749 Zhang, Y., Proenca, R., Maffei, M., Barone, M., Leopold, L., and Friedman, J.M. (1994).

750 Positional cloning of the mouse obese gene and its human homologue. Nature 372 ,

$751 \quad 425-432$. 


\section{Table $\mathbf{1}$ (on next page) \\ DEG Morphant Vs. Control}

Top-50 differentially expressed genes ranked by adjusted p.value from lepamorphants compared to control. LogFC $=\log _{2}$ fold change. 

control.

\begin{tabular}{|c|c|c|c|}
\hline SYMBOL & $\log \mathrm{FC}$ & adj.P.Val & ENTREZ ID \\
\hline CABZ01080435.1 & 4.060502 & $1.24 \mathrm{E}-06$ & NA \\
\hline LOC100537029 & 3.818306 & $1.24 \mathrm{E}-06$ & 100537029 \\
\hline LOC799595 & 3.276172 & $1.24 \mathrm{E}-06$ & NA \\
\hline igsf21b & -2.2581 & $1.24 \mathrm{E}-06$ & 567714 \\
\hline LOC557824 & 1.890801 & $1.92 \mathrm{E}-06$ & NA \\
\hline nes & 2.455737 & 2.19E-06 & 100150939 \\
\hline$d l b$ & 1.808859 & 2.32E-06 & 30141 \\
\hline pou2f2a & 1.60189 & $2.70 \mathrm{E}-06$ & 557055 \\
\hline insm1a & 1.767329 & $3.42 \mathrm{E}-06$ & 402941 \\
\hline notch1a & 2.04673 & $3.61 \mathrm{E}-06$ & 30718 \\
\hline sagb & -2.24925 & 4.11E-06 & 619268 \\
\hline si:dkey-204/11.1 & 2.446131 & 4.73E-06 & 100006301 \\
\hline$t t c 7 a$ & -1.1399 & 4.73E-06 & 559345 \\
\hline LOC100537727 & 1.920483 & $5.15 \mathrm{E}-06$ & NA \\
\hline gngt $2 b$ & -4.75024 & 5.17E-06 & 797361 \\
\hline gng $13 b$ & -1.85747 & 5.79E-06 & 436673 \\
\hline dld & 1.648308 & 6.62E-06 & 30138 \\
\hline dnajb1b & 1.81376 & $6.95 \mathrm{E}-06$ & 327244 \\
\hline iars & 2.086352 & 8.43E-06 & 334393 \\
\hline CTBP2 & -1.63952 & 8.78E-06 & NA \\
\hline opn1mw1 & -6.1018 & 8.78E-06 & 30503 \\
\hline$g n b 3 b$ & -3.928 & 9.69E-06 & 406483 \\
\hline LOC561947 & 1.846519 & $9.83 \mathrm{E}-06$ & NA \\
\hline LOC100329434 & 1.596356 & 9.83E-06 & NA \\
\hline LOC568543 & -1.34136 & $9.83 \mathrm{E}-06$ & NA \\
\hline foxn4 & 2.188575 & $1.01 \mathrm{E}-05$ & 30315 \\
\hline dla & 2.058014 & 1.03E-05 & 30131 \\
\hline LOC100536392 & -2.02991 & $1.03 \mathrm{E}-05$ & NA \\
\hline$t m \times 3$ & -2.25409 & $1.03 \mathrm{E}-05$ & 553578 \\
\hline si:ch211-88n13.3 & -2.31863 & 1.03E-05 & NA \\
\hline prph2b & -4.08143 & 1.07E-05 & 559209 \\
\hline gnb3a & -2.24428 & 1.13E-05 & 436710 \\
\hline brf1a & 2.034817 & 1.17E-05 & 334402 \\
\hline tab1 & 1.357627 & 1.17E-05 & 403084 \\
\hline opn1/w2 & -4.04306 & 1.17E-05 & 436716 \\
\hline LOC569340 & -2.80272 & $1.22 \mathrm{E}-05$ & 569340 \\
\hline LOC100331226 & -1.8585 & 1.29E-05 & NA \\
\hline neurog1 & 1.919667 & $1.38 \mathrm{E}-05$ & 30239 \\
\hline LOC559232 & -2.30088 & $1.48 \mathrm{E}-05$ & NA \\
\hline cacna2d4b & -1.85537 & 1.82E-05 & 100150428 \\
\hline LOC570404 & 3.754077 & 1.97E-05 & NA \\
\hline LOC562934 & -1.23679 & 2.01E-05 & NA \\
\hline arr3a & -3.38027 & 2.01E-05 & 436678 \\
\hline nelfa & 2.257915 & 2.03E-05 & 559677 \\
\hline hbbe3 & 2.846799 & 2.26E-05 & 30596 \\
\hline zgc:73359 & -3.65251 & 2.37E-05 & 393810 \\
\hline$c x c l-c 1 c$ & 2.146952 & 2.51E-05 & NA \\
\hline arhgef1b & 1.687267 & 2.51E-05 & 557983 \\
\hline si:ch1073-303/5.1 & 1.40302 & $2.51 \mathrm{E}-05$ & NA \\
\hline $\operatorname{lin} 28 a$ & 1.191522 & 2.51E-05 & 394066 \\
\hline
\end{tabular}




\section{Table 2 (on next page)}

Top DEG Rescue Vs. Morphant

Top-50 differentially expressed genes ranked by adjusted p.value from leparescue compared to lepamorphants. $\operatorname{LogFC}=\log _{2}$ fold change. 

morphants.

\begin{tabular}{|c|c|c|c|}
\hline SYMBOL & $\log F C$ & adj.P.Val & ENTREZ ID \\
\hline SI:DKEY-24I24.3 & 3.551056 & 3.87E-09 & NA \\
\hline$v w f$ & 3.524634 & 3.49E-06 & 570643 \\
\hline LOC568400 & 2.24809 & 8.17E-06 & NA \\
\hline LOC570208 & 1.540514 & 8.19E-06 & NA \\
\hline or125-2 & 2.928568 & 2.24E-05 & 100150140 \\
\hline LOC793072 & 2.752483 & $2.24 \mathrm{E}-05$ & NA \\
\hline or115-6 & 2.690795 & $2.24 \mathrm{E}-05$ & 678539 \\
\hline LOC100535281 & 2.679196 & 2.24E-05 & NA \\
\hline si:ch211-237a4.2 & 2.352812 & $2.24 \mathrm{E}-05$ & 100034537 \\
\hline si:dkey-28d5.10 & 2.216788 & $2.24 \mathrm{E}-05$ & 799800 \\
\hline or125-4 & 1.839774 & $2.24 \mathrm{E}-05$ & 100148706 \\
\hline$c d k 11 b$ & -1.58101 & $2.24 \mathrm{E}-05$ & 494103 \\
\hline LOC100333199 & -1.59951 & $2.24 \mathrm{E}-05$ & NA \\
\hline SOS1 & -1.61741 & 2.24E-05 & NA \\
\hline plxnd1 & -1.7363 & $2.24 \mathrm{E}-05$ & 402998 \\
\hline IGHV2-2 & 2.245677 & 2.89E-05 & NA \\
\hline LOC794788 & 2.231972 & 2.89E-05 & NA \\
\hline LOC100333311 & -1.72142 & $3.12 \mathrm{E}-05$ & NA \\
\hline$d e p d c 1 b$ & -2.00778 & 4.36E-05 & 100006170 \\
\hline zgc:158376 & -1.36533 & $4.52 \mathrm{E}-05$ & 100101643 \\
\hline dph6 & -1.42828 & 4.73E-05 & 503603 \\
\hline$b c / 2$ & -1.40772 & $6.54 \mathrm{E}-05$ & NA \\
\hline galnt10 & -1.42208 & $6.54 \mathrm{E}-05$ & 767665 \\
\hline LOC100007376 & 1.217926 & 7.14E-05 & 100007376 \\
\hline LOC570185 & 2.012359 & 7.71E-05 & 570185 \\
\hline adrb1 & 2.119886 & 8.64E-05 & 557194 \\
\hline si:ch211-160j14.2 & 1.438866 & 8.88E-05 & 792922 \\
\hline LOC799771 & -1.23956 & 9.23E-05 & NA \\
\hline LOC100534901 & 2.382595 & $9.48 \mathrm{E}-05$ & NA \\
\hline LOC100536834 & 1.848393 & $9.48 \mathrm{E}-05$ & 100536834 \\
\hline pou2f2a & -1.3119 & $9.48 \mathrm{E}-05$ & 557055 \\
\hline$B \times 936386.1$ & 1.247975 & 0.000113 & NA \\
\hline slitrk5 & -1.37301 & 0.000137 & 100330023 \\
\hline LOC100149966 & -2.27763 & 0.000137 & 100149966 \\
\hline taar10c & 2.26676 & 0.000138 & 794440 \\
\hline$c d c 42 b p b$ & -1.18873 & 0.000138 & 567039 \\
\hline si:rp71-56i13.6 & -2.08428 & 0.000138 & 564457 \\
\hline USP30 & -2.15151 & 0.000141 & NA \\
\hline or106-2 & 1.17958 & 0.000148 & 100861459 \\
\hline LOC100536940 & 2.318071 & 0.000154 & NA \\
\hline CXCL11 & 2.504981 & 0.000162 & NA \\
\hline zgc:85975 & -1.77849 & 0.000166 & 406549 \\
\hline$f z d 5$ & -1.2611 & 0.000167 & 30364 \\
\hline LOC100535489 & 2.303531 & 0.000167 & NA \\
\hline polh & -1.14961 & 0.000203 & 678520 \\
\hline SI:DKEY-256/11.6 & 1.098104 & 0.000217 & NA \\
\hline zbtb16a & -1.49196 & 0.000217 & 323269 \\
\hline LOC100331199 & 2.513658 & 0.000231 & NA \\
\hline LOC560659 & 2.383395 & 0.000231 & 560659 \\
\hline trim35-3 & 1.341874 & 0.000231 & 100003935 \\
\hline
\end{tabular}




\section{Table 3(on next page)}

Top DEG Rescue Vs. Control

Top-50 differentially expressed genes ranked by adjusted p.value from leparescue compared to control. $\log F C=\log _{2}$ fold change. 
1 Table 3: Top-50 differentially expressed genes from lepa rescue compared to control.

\begin{tabular}{|c|c|c|c|}
\hline SYMBOL & $\log F C$ & adj.P.Val & ENTREZ ID \\
\hline SI:DKEY-24I24.3 & 3.599239 & $3.26 \mathrm{E}-10$ & NA \\
\hline$v w f$ & 3.385689 & $6.70 \mathrm{E}-07$ & 570643 \\
\hline LOC796392 & -1.60622 & $6.70 \mathrm{E}-07$ & NA \\
\hline LOC570208 & 1.538632 & $6.75 \mathrm{E}-07$ & NA \\
\hline LOC100149028 & -1.87278 & $6.75 \mathrm{E}-07$ & NA \\
\hline pank2 & -2.25072 & $6.75 \mathrm{E}-07$ & 570866 \\
\hline LOC793072 & 2.935593 & 1.06E-06 & NA \\
\hline or115-6 & 2.933138 & 1.06E-06 & 678539 \\
\hline LOC568400 & 2.075418 & 1.06E-06 & NA \\
\hline or125-2 & 2.962312 & 1.34E-06 & 100150140 \\
\hline or125-4 & 1.906985 & $1.40 \mathrm{E}-06$ & 100148706 \\
\hline parp6a & -1.64685 & $1.40 \mathrm{E}-06$ & 436810 \\
\hline si:ch211-243a15.1 & -1.77679 & $1.40 \mathrm{E}-06$ & 100005033 \\
\hline map3k12 & -1.82325 & $1.40 \mathrm{E}-06$ & 404626 \\
\hline IGHV2-2 & 2.409117 & $1.42 \mathrm{E}-06$ & NA \\
\hline LOC100536180 & 2.170446 & 1.66E-06 & NA \\
\hline SI:DKEY-256/11.6 & 1.448259 & 1.66E-06 & NA \\
\hline slitrk5 & -1.67491 & 1.93E-06 & 100330023 \\
\hline LOC569340 & -3.34108 & 1.93E-06 & 569340 \\
\hline BX936386.1 & 1.488632 & 1.97E-06 & NA \\
\hline LOC100535281 & 2.606151 & 2.21E-06 & NA \\
\hline si:dkey-28d5.10 & 2.068829 & 2.21E-06 & 799800 \\
\hline TMPO & 1.986495 & 2.21E-06 & NA \\
\hline LOC562934 & -1.51774 & 2.21E-06 & NA \\
\hline LOC568961 & -1.74594 & 2.21E-06 & NA \\
\hline LOC794788 & 2.211161 & $2.56 \mathrm{E}-06$ & NA \\
\hline CTBP2 & -1.74604 & 2.56E-06 & NA \\
\hline LOC100332615 & -2.10437 & $2.56 \mathrm{E}-06$ & 100332615 \\
\hline si:ch211-237a4.2 & 2.20162 & $3.12 \mathrm{E}-06$ & 100034537 \\
\hline$c d h 23$ & -2.03904 & $3.20 \mathrm{E}-06$ & 407978 \\
\hline LOC100536867 & 1.858425 & 3.81E-06 & 100536867 \\
\hline sc:d0343 & -1.59675 & 3.81E-06 & NA \\
\hline LOC558743 & -1.42439 & 4.01E-06 & NA \\
\hline zgc:123060 & -1.89975 & 4.07E-06 & 641487 \\
\hline LOC100536834 & 1.974369 & 4.33E-06 & 100536834 \\
\hline LOC570185 & 2.056828 & 4.91E-06 & 570185 \\
\hline ankrd13b & -1.22985 & 4.91E-06 & 568981 \\
\hline Irrtm1 & -1.26398 & 4.91E-06 & 570385 \\
\hline arvcfa & -1.43558 & 4.91E-06 & 572216 \\
\hline$d d r 2$ & -1.55141 & 4.91E-06 & NA \\
\hline Irit1b & -2.51937 & 4.91E-06 & 553432 \\
\hline LOC560659 & 2.755338 & 5.11E-06 & 560659 \\
\hline LOC100006857 & -1.38684 & $5.45 \mathrm{E}-06$ & NA \\
\hline $\operatorname{lin} 28 a$ & 1.338285 & 5.56E-06 & 394066 \\
\hline sagb & -1.99538 & $5.65 \mathrm{E}-06$ & 619268 \\
\hline igsf21b & -1.70889 & $6.12 \mathrm{E}-06$ & 567714 \\
\hline LOC555422 & -2.11608 & $6.12 \mathrm{E}-06$ & 555422 \\
\hline$n r 1 d 1$ & -3.2824 & $6.12 \mathrm{E}-06$ & 494487 \\
\hline LOC100333199 & -1.33657 & $6.14 \mathrm{E}-06$ & NA \\
\hline zgc:111992 & 1.544128 & $6.26 \mathrm{E}-06$ & NA \\
\hline
\end{tabular}




\section{Table 4 (on next page)}

KEGG Pathway Enrichment All Pairwise Comparisons

Kyoto Encyclopedia of Genes and Genomes (KEGG) pathway enrichment $(P<0.05)$ for 3

separate pairwise comparisons: I. lepaknockdown compared to control (MO::WT), II. leparescue compared to lepaknockdown (RS::MO), and III. leparescue compared to control (RS::WT). 
1 Table 4: KEGG Pathway enrichment analyses from each comparison $(P<0.05)$. DEG's pertaining to each KEGG

2 pathway are appended in the supplementary gene list.

\begin{tabular}{|c|c|c|c|}
\hline & KEGG Pathway (<0.05); LEPA Rescue - Control (3,036 Entrez ID's; 5,105 DEG's) & $\begin{array}{l}\text { Adj. } \\
\text { p.value }\end{array}$ & DEG Count \\
\hline dre04080 & Neuroactive ligand-receptor interaction & 4.63E-07 & 94 \\
\hline dre04070 & Phosphatidylinositol signaling system & 2.83E-03 & 29 \\
\hline dre04744 & Phototransduction & 9.92E-03 & 13 \\
\hline \multirow[t]{2}{*}{ dre00534 } & Glycosaminoglycan biosynthesis - heparan sulfate / heparin & $1.50 \mathrm{E}-02$ & 11 \\
\hline & KEGG Pathway (<0.05); LEPA Rescue - LEPA Morphant (999 Entrez gene ID's) & $\begin{array}{c}\text { Adj. } \\
\text { p.value }\end{array}$ & DEG Count \\
\hline \multirow[t]{2}{*}{ dre04330 } & Notch signaling pathway & 0.000108 & 12 \\
\hline & KEGG Pathway (< 0.05); LEPA Morphant - Control (970 Entrez ID's) & $\begin{array}{c}\text { Adj. } \\
\text { p.value }\end{array}$ & DEG Count \\
\hline dre04744 & Phototransduction & 2.03E-08 & 14 \\
\hline dre03008 & Ribosome biogenesis in eukaryotes & $6.65 \mathrm{E}-03$ & 12 \\
\hline dre04330 & Notch signaling pathway & 1.08E-02 & 10 \\
\hline dre04080 & Neuroactive ligand-receptor interaction & $2.16 \mathrm{E}-02$ & 34 \\
\hline
\end{tabular}




\section{Figure 1}

Differentially expressed genes for all datasets

Volcano plots $(1 \mathrm{~A}-1 \mathrm{C})$ represent the adjusted p.value versus fold change for each gene across three pairwise comparisons (listed above each plot). Each point represents one gene.

Differentially expressed genes are teal; red points denote genes that do not meet differentially expressed selection criterions. Venn diagram (1D) represents the number of differentially expressed genes (DEG's) in each comparison at adjusted p.value $<0.01$ and $\log _{2}$ fold change $<-0.5$ or $>0.5$. "morphant - control" refers to genes that are differentially expressed in lepa knockdown zebrafish compared to uninjected controls. "rescue morphant" refers to genes that are differentially expressed in lepa rescue zebrafish compared to lepa knockdown. "rescue - control" refers to genes that are differentially expressed in lepa rescue zebrafish compared to uninjected controls. 
$1 \mathrm{~A}$

\section{LEPA Knockdown Control}

Not.Significant - Differentially.Expressed
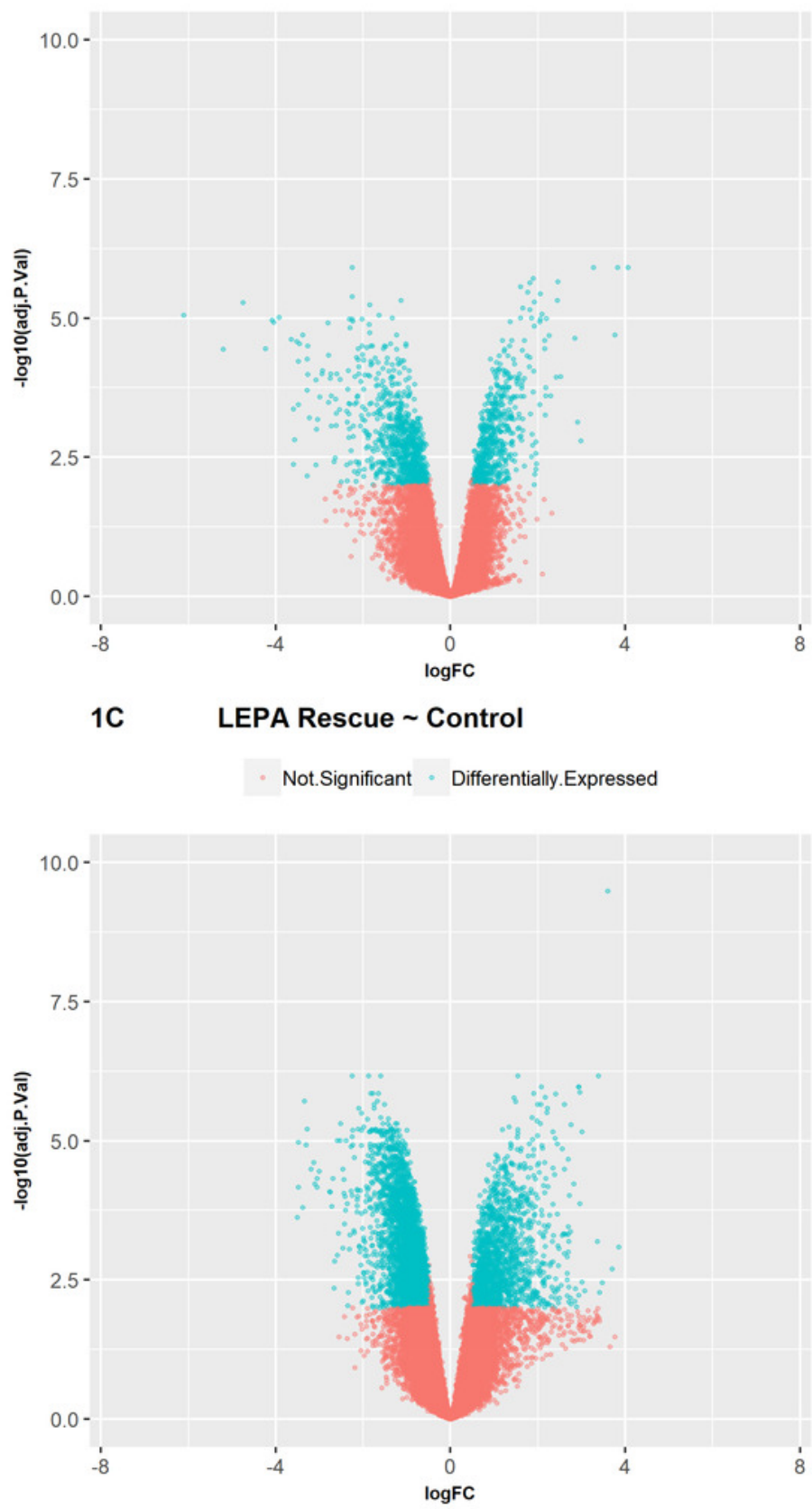

1B

\section{LEPA Rescue LEPA Knockdown}

Not.Significant - Differentially.Expressed

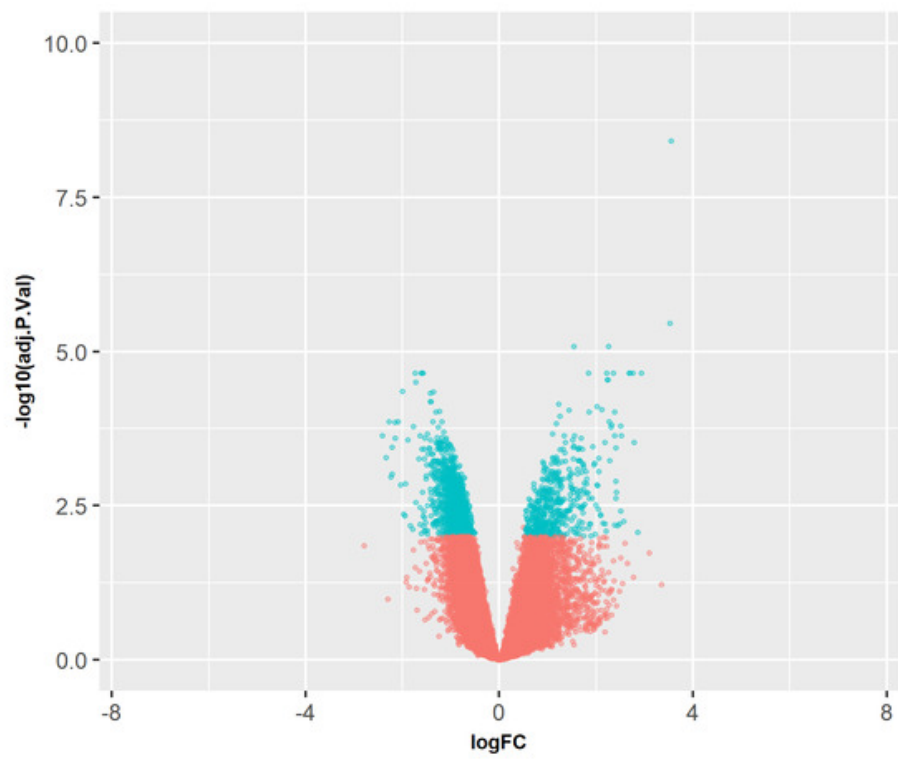

1D Differentially Expressed Genes

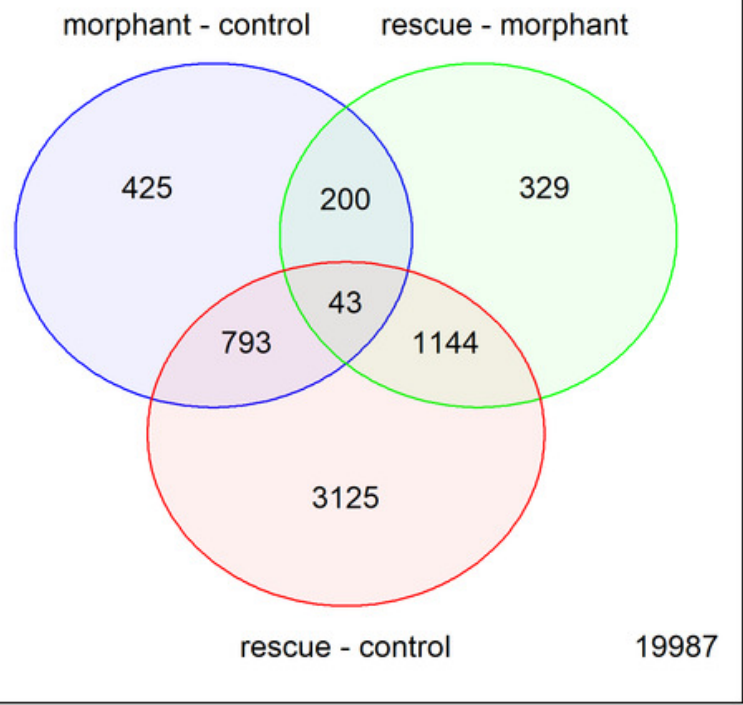


Figure 2

Neuractive Ligand-Receptor Interactions

(Kyoto Encyclopedia of Genes and Genomes (KEGG) pathway enrichment $(P<0.05)$

representing neuroactive ligand-receptor interactions from leparescue compared to control microarrays. Color scale reflects $\log _{2}$ fold change for differentially expressed genes.

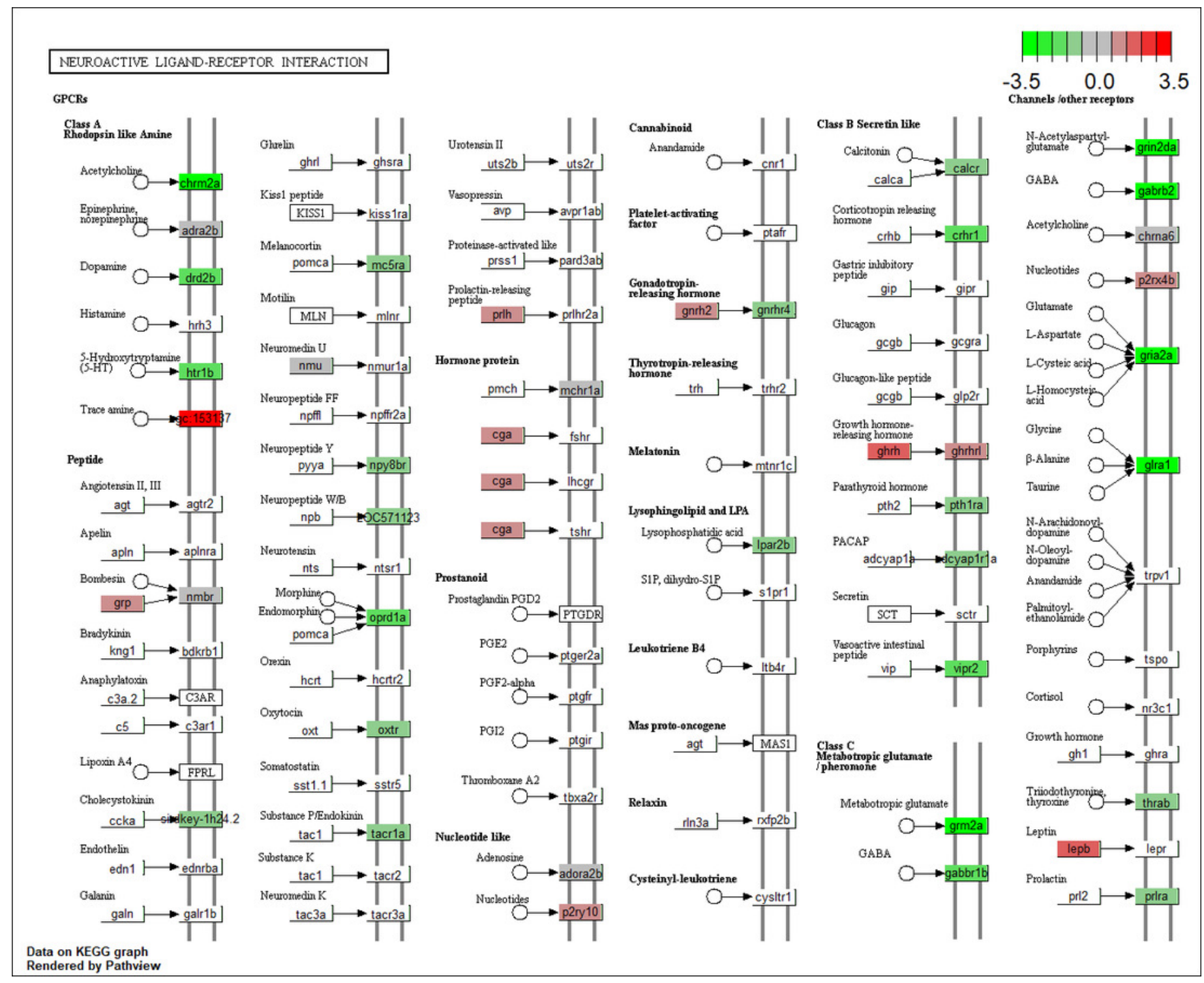


Figure 3

Phosphatidyl Inositol Signaling

Kyoto Encyclopedia of Genes and Genomes (KEGG) pathway enrichment $(P<0.05)$

representing phosphatidylinositol signaling from leparescue compared to control microarrays. Color scale reflects $\log _{2}$ fold change for differentially expressed genes.

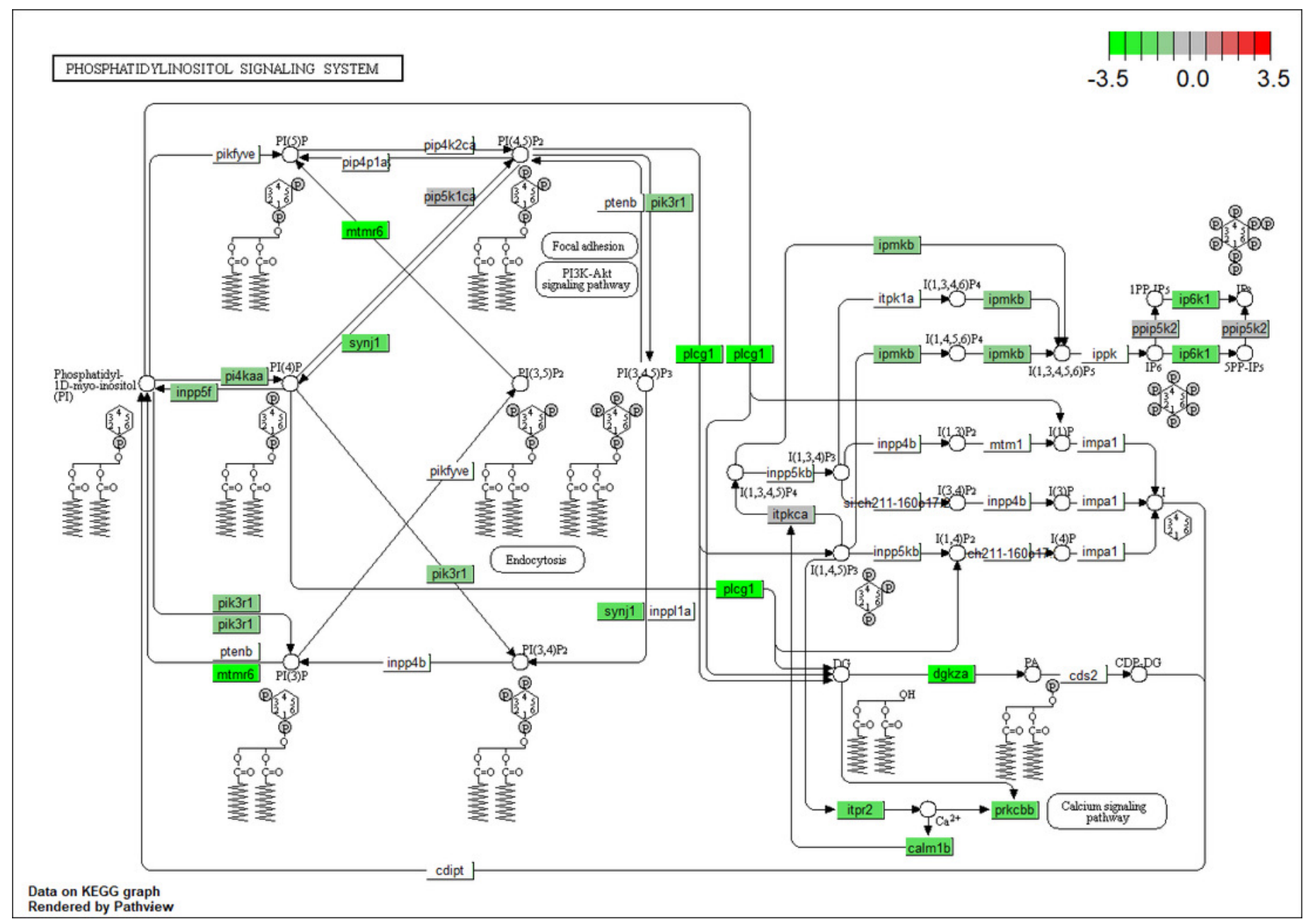




\section{Figure 4}

Top-Ranked DEG's (A) and Leptin Signaling DEG's

A) Heatmap displaying the top-50 ranked DEG's in lepa morphants compared to controls.

Genes (rows) are clustered to represent Delta/Notch genes (orange), Phototransduction

(purple), or grey (other). (B) Heatmap containing the zebrafish homologs of leptin signaling genes identified by Park and Ahima (2014), including lepa, lepb, lepr, LOC794738, LOC100537326, akt3, pik3r1, prkaa2, socs2, socs5b, socs9, npy8br, npy8ar, mc5ra, foxo1b, jak2a, jak2b, camkk2, map3k12, agrp2, and cart1. Genes displaying large fold changes (vwf, opn1/w1, igsf21b, LOC100537029), and others that regulate lipid (acacb, dagla, daglb, Ipl, crot, plin1, cpt1b, pank2) or RNA metabolism (smg5, dicer1, LOC796505, LOC570775) are also included.. Each gene (row) is color coded to represent high (red) and low (green) levels of expression. Columns are color coded by treatment - blue (lepa rescue), red (lepa morphants), green (uninjected controls). 


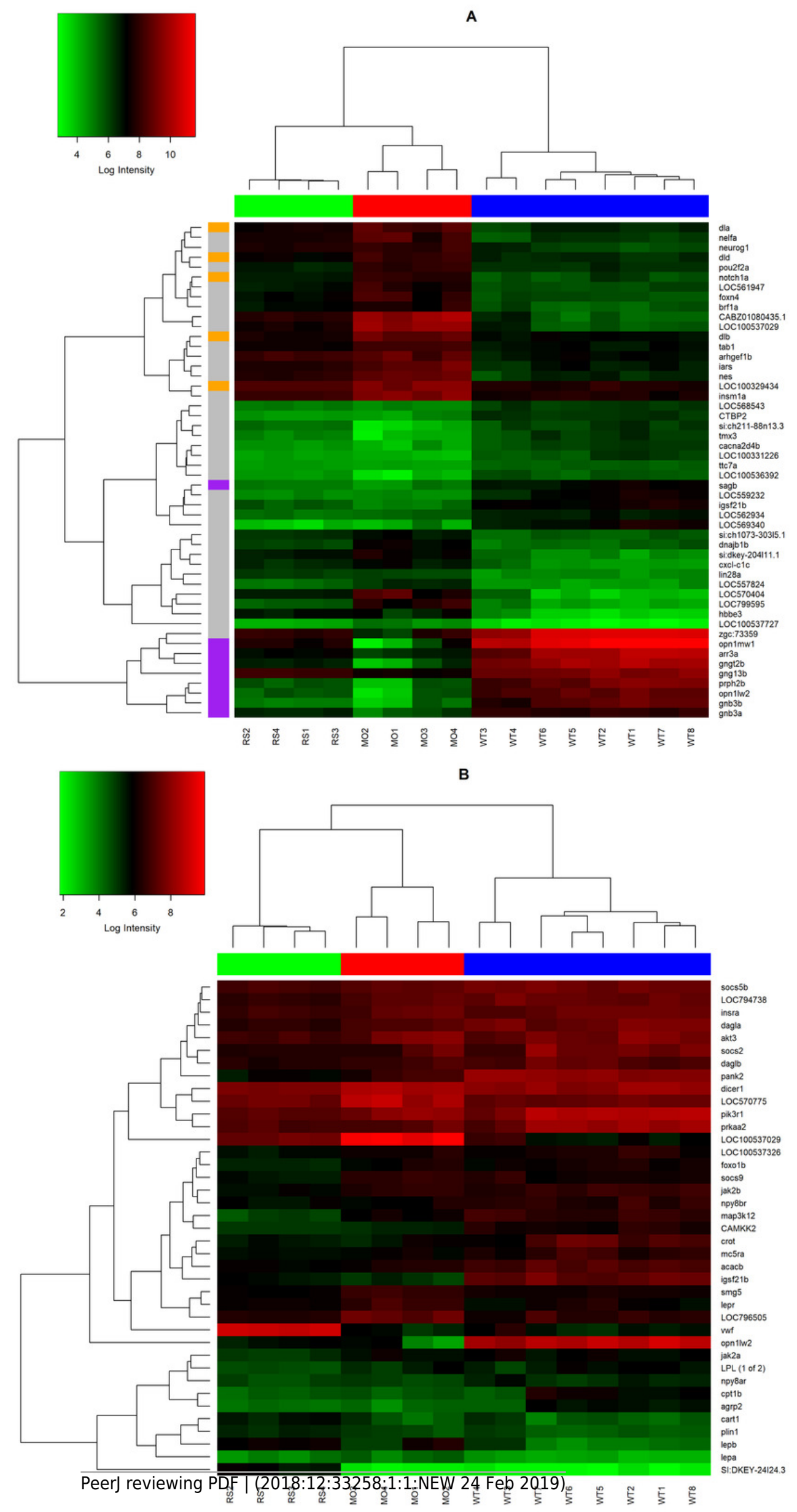


Figure 5

GO Analyses Morphant Vs. Control

Gene Ontology biological process enrichment results $(P<0.01)$ from lepa morphants

compared to control; the barplot contains 54 enriched terms. Color scale reflects adjusted

p.value; the $\mathrm{x}$-axis denotes the number of DEG's that map to each enriched term.

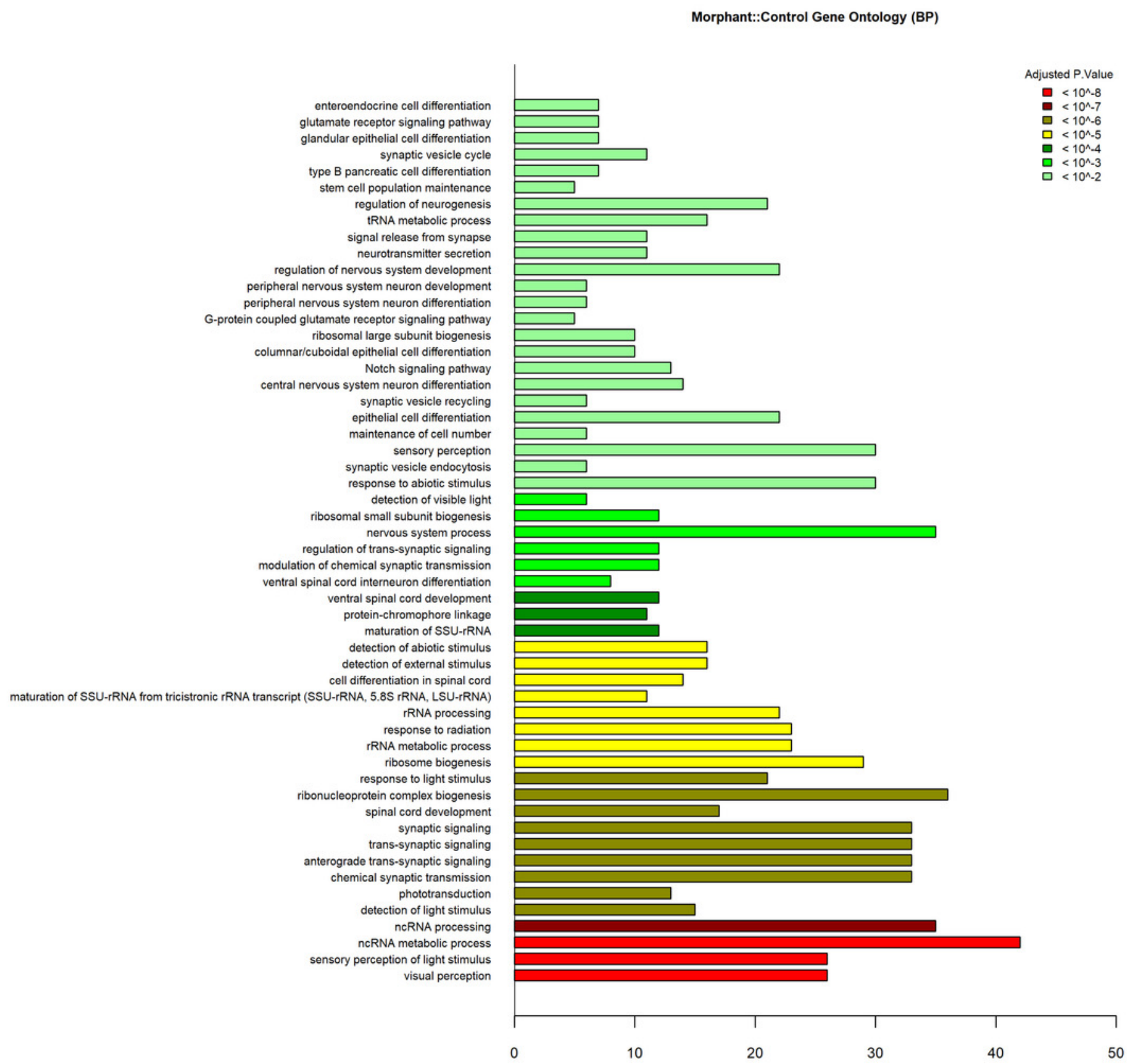




\section{Figure 6}

GO Analyses Rescue Vs. Morphants

Gene Ontology enrichment results $(P<0.01)$ containing $n=22$ terms derived from all three categories (biological process, cellular component, and molecular function) in lepa rescue compared to lepa morphant treatments. Color scale reflects adjusted p.value; the $\mathrm{x}$-axis denotes the number of DEG's that map to each enriched term. 
Rescue::Morphant Gene Ontology (BP,MF,CC)

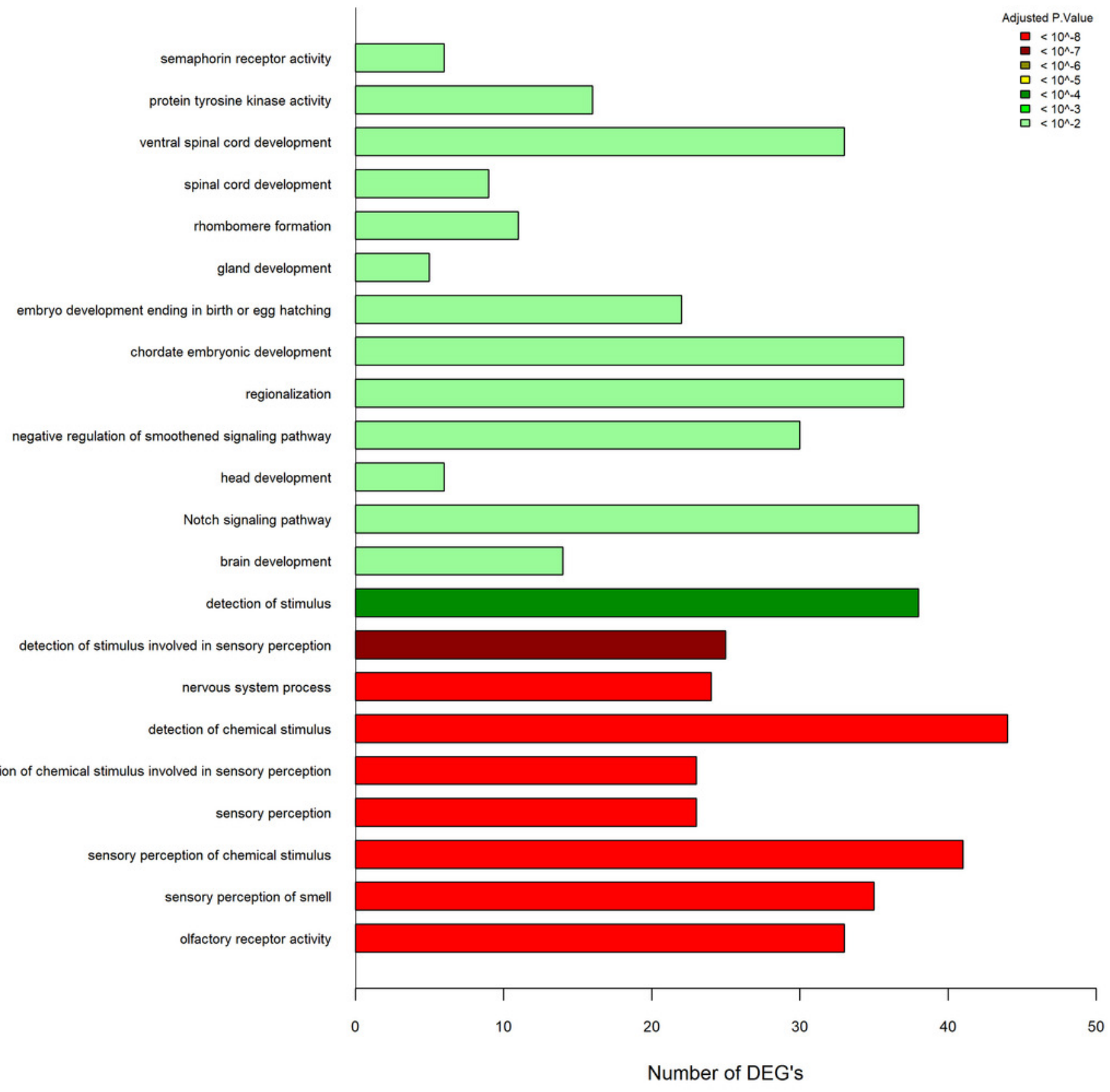




\section{Figure 7}

\section{GO Analyses Rescue Vs. Control}

Gene Ontology biological process enrichment results $(P<0.01)$ from lepa rescue compared to control treatments representing $n=39$ terms. Color scale reflects adjusted p.value; the $x$ axis denotes the number of DEG's that map to each enriched term.

Rescue::Control Gene Ontology(BP)

regulation of small GTPase mediated signal transduction signal release from synapse neurotransmitter secretion endocytosis protein homooligomerization STAT cascade JAK-STAT cascade regulation of voltage-gated calcium channel activity detection of visible light postsynapse organization synapse organization vesicle-mediated transport in synapse phototransduction synaptic vesicle cycle postsynaptic membrane organization detection of abiotic stimulus detection of external stimulus response to abiotic stimulus detection of light stimulus regulation of trans-synaptic signaling modulation of chemical synaptic transmission response to radiation synaptic vesicle recycling sensory perception of light stimulus response to light stimulus visual perception synaptic vesicle endocytosis detection of chemical stimulus detection of chemical stimulus involved in sensory perception detection of stimulus involved in sensory perception detection of stimulus trans-synaptic signaling anterograde trans-synaptic signaling chemical synaptic transmission sensory perception of smell synaptic signaling sensory perception of chemical stimulus nervous system process sensory perception
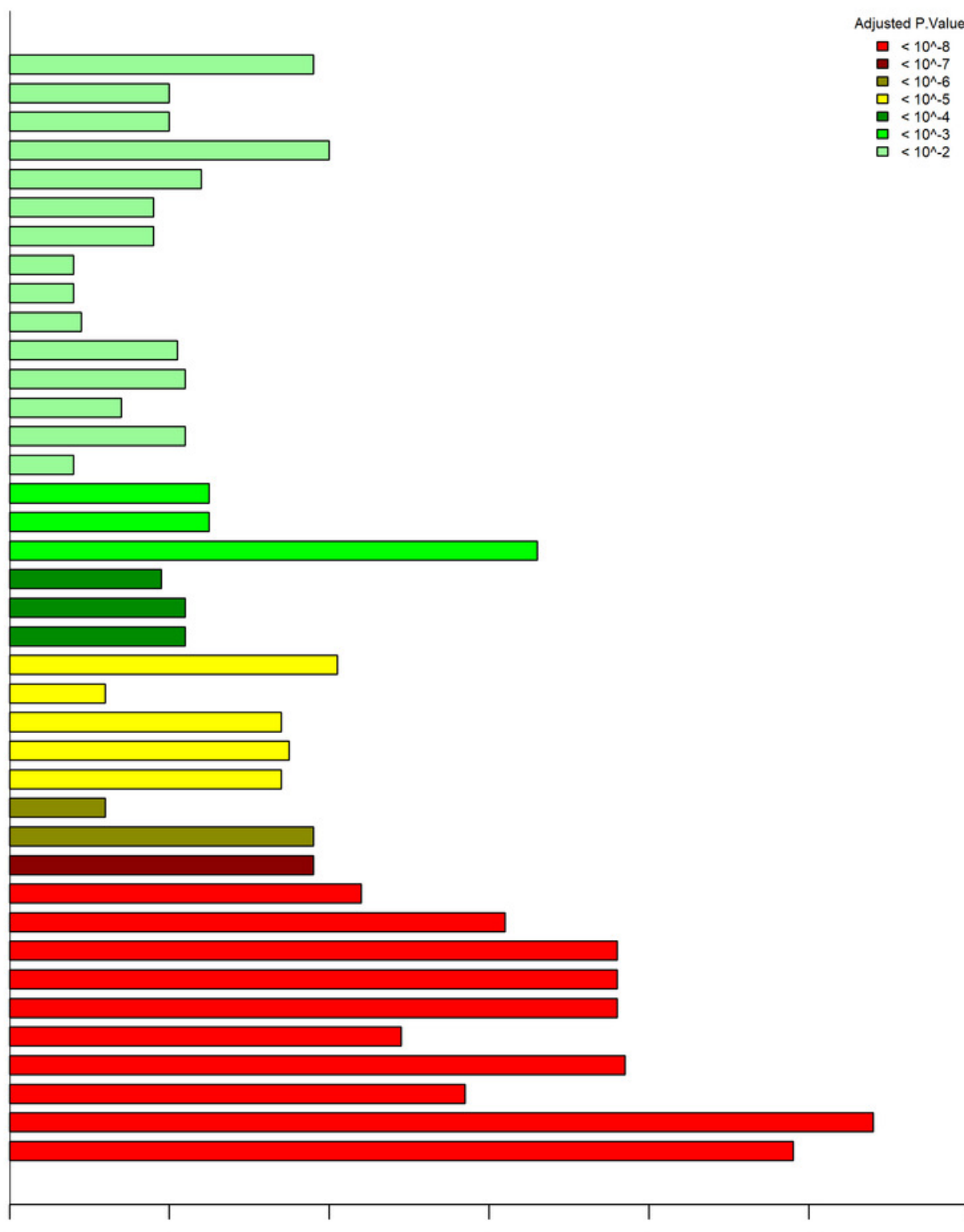

0

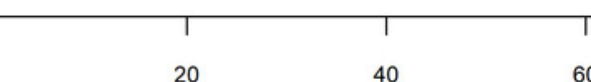

